\title{
Truth-tracking judgment aggregation over interconnected issues
}

\author{
Irem Bozbay*
}

November 2018

\begin{abstract}
This paper analyzes the problem of aggregating individual judgments over two interconnected issues. Voters share a common preference which is state-dependent, but they hold private information about what the state might be. I assume strategic voting in a Bayesian voting game setting and I want to determine voting rules which induce an efficient Bayesian Nash equilibrium in truthful strategies, hence lead to collective judgments that efficiently incorporate all private information. Interconnectedness may lead to private information that is inconsistent with the state, which leads to the impossibility of efficient information aggregation. Once I introduce the possibility of abstention, the negative conclusion no longer prevails and there is always a voting rule which aggregates information efficiently. I obtain a similar positive result when I rule out the possibility of inconsistent private information. I analyze the situations in which such rules exist whenever necessary, as well as the nature of these rules.

Keywords: judgment aggregation, efficient information aggregation, strategic voting, private information
\end{abstract}

JEL Classification Numbers: C70, D70, D71, D80, D82

\section{Introduction}

How should a group of individuals form a collective 'yes' or 'no' judgment on several issues given judgments of the group members? Judgment aggregation theory focuses on this question which has wide applications in many collective decision-making bodies, ranging from expert panels to juries, legislative committees to multi-member courts and so on. A typical example is the problem of the jury in a court trial, where the jury needs to form a collective 'yes' or 'no' judgment on whether the defendant has broken the contract and whether the contract is legally valid. The collective judgments on these issues typically determine a third issue, whether the defendant should be convicted. The issues for which a vote is taken can in principle be 'mutually interconnected' in such problems, i.e., a judgment made on one issue may restrict the

\footnotetext{
*University of Surrey. E-mail: I.Bozbay@surrey.ac.uk.
} 
judgment on the other. For instance, an expert panel might need to form 'yes' or 'no' judgments on the two issues of whether the $\mathrm{CO}_{2}$ emissions are above a given threshold and whether there will be a critical temperature change, where a 'yes' judgment on the first issue requires a 'yes' judgment on the second issue; the United Nations security council might need to form judgments on whether a country will suffer a civil war, and whether it will suffer a severe socio-economic crisis, where the former implies the latter; the EU commission might need to form judgments on whether a candidate country has implemented benchmark political criteria and whether the country's economy is unstable, where the negation of the former implies the latter and so on. As in the court trial example where the jury convicts the defendant or not, groups may take an action depending on the collective judgments, such as a large scale intervention in the UN example or providing additional funds to the candidate country in the EU commission example.

This paper considers a judgment aggregation problem where there are two interconnected issues in the agenda of a committee. I assume that there is an objective truth to be found, called the state (of the world). Individuals share a common preference for state-matching (true) collective judgments, but hold possibly conflicting private information about the state. I assume strategic voting, that is, rational behavior in the sense of a Bayesian Nash equilibrium of the corresponding game and I want to answer the following question: which voting rules (if any) lead to collective judgments which are efficient given all voters' private information? Such voting rules would give incentives for truthful voting behavior in equilibrium, which cannot be taken for granted even when voters have no conflict of interest. That's because a voter can change the collective judgment on an issue only when she is pivotal, and being pivotal may eliminate the strategic incentive to vote truthfully. This observation is due to Austen-Smith and Banks (1996), where they study binary collective choice problems (which can be seen as a judgment aggregation problem with only one issue) with common interests and private information, and it gives rise to the analysis and design of voting rules which lead to truthful behavior of individuals as well as correct decisions.

The information aggregation analysis has been applied to multiple issues previously in Ahn and Oliveros (2013), Bozbay, Dietrich and Peters (2014) and De Clippel and Eliaz (2015). While the first and the last papers compare the asymptotic efficiency of different mechanisms, the second studies the problem of designing a voting rule, as in the present paper. These papers do not consider logical interconnections between issues and they assume independent issues. ${ }^{1}$ Given that interconnections are an important aspect of judgment aggregation theory and they are important in practice, this paper studies efficient information aggregation for agendas with two interconnected issues, where the issues present a particular logical structure and judgments made

\footnotetext{
${ }^{1}$ Note that analysing multiple issues in the absence of interconnections does not reduce to analysing each issue separately since preferences are non-separable and they establish links between issues. For instance, in the above mentioned papers, a voter's utility is not necessarily monotonic in the number of accepted issues. On the other hand, interconnections are what make judgment aggregation nontrivial in the absence of strategic behavior.
} 
on issues logically constrain each other. In the absence of private information and the strategic incentives that follow, interconnectedness may lead to logically inconsistent collective judgments (as reviewed in the next section) due to procedural requirements on the voting rule. In the present paper's framework, interconnectedness may lead to private information that is inconsistent with the state which leads to the (partial) impossibility of efficient information aggregation. While with multiple independent issues and with a single issue, a voting rule which induces an efficient Bayesian Nash equilibrium in truthful strategies always exists (see Austen-Smith and Banks, 1996 and Bozbay, Dietrich and Peters, 2014), I find that with two interconnected issues such voting rule exists only under a strong condition relating the model parameters and preferences. I derive a similar result for a variety of definitions of truthful (or, informative) voting in Section 3. These results are mainly driven by the difficulty of truthfully revealing the private information that is inconsistent with the state. The possibility of inconsistent private information naturally leads to a mismatch between the state space and the type space, which eventually drives the impossibility results. ${ }^{2}$ Once this mismatch is ruled out by assuming that private information is distributed jointly rather than independently for issues, it is possible to obtain a general existence result, as I show in Section 5.

Can inconsistent private information always be avoided in reality? If the evidence for each issue comes from a different expert (or data set, witness, experiment, etc.) and experts act independently, one may end up with overall inconsistent information when issues are interconnected. The problem becomes increasingly relevant the larger the set of available information sources is, which is a reality of our times. To overcome the impossibility in the case of inconsistent private information, I introduce abstention as a possible voting behavior in Section 4. Indeed, Feddersen and Pesendorfer (1996) show that less informed (or uninformed) voters have an incentive to delegate their vote via abstention to more informed voters in a model where (a fraction of) voters hold state-dependent common preferences and private information. In the present paper's framework, a voter with inconsistent private information knows that she is 'ill-informed'. As preferences are common, this voter might prefer to abstain in the hope that voters without inconsistent information will determine the decision. I consider the particular voting behavior (which I call informative voting with abstention) where each voter with the inconsistent type abstains while others truthfully report. I show that one can always find a voting rule which guarantees an efficient Bayesian Nash equilibrium in such voting behavior. Apart from the general existence claim, I also focus on the specific kinds of common preferences. Under simple preferences where state-matching decisions are preferred to non-matching ones, it will turn out that one should use a 'quota rule with exception', which behaves like a quota rule ${ }^{3}$

\footnotetext{
${ }^{2}$ In that sense, Section 3 of this paper contributes to the literature which studies the effects of relative richness of signal and state spaces to information aggregation; see, e.g., Schmitz and Tröger (2012), Barelli, Bhattacharya and Siga (2018) and Bozbay and Peters (2018). In each of these papers, an efficient Bayesian Nash equilibrium in truthful strategies does not generally exist, though each considers a very different model than the current paper's.

${ }^{3} \mathrm{~A}$ quota rule decides on each issue according to whether the number of 'yes' judgments on the issue exceeds a particular quota.
} 
except when neither issue reaches the acceptance quota. In that case, the rule decides according to an 'exception rule'. Next, I focus on consequentialist preferences, which assume that group decision on issues leads to group action (such as convicting or acquitting a defendant or a large scale UN intervention) and a voter prefers correct group action to incorrect one regardless of the correctness of the underlying decision. Under consequentialist preferences, although a voting rule which efficiently aggregates information always exists, it is a quota rule with exception only under certain restrictions on the parameters of the model.

The paper proceeds as follows. In Section 2, I introduce the model. Section 3 addresses the key question of whether a voting rule that leads to efficient decisions as well as truthful voting behavior in equilibrium exists. The answer is no in most cases, and under a variety of possible definitions of truthful voting. In Sections 4 and 5, I propose the possibility of abstaining and jointly distributed private information respectively, as possible escapes from the impossibility. Sections 3,4 and 5 all analyze the particular kinds of truth-tracking preferences (simple and consequentialist) as well as the general case. All proofs are given in the Appendix.

\subsection{Related Literature}

The judgment aggregation literature contains several possibility and impossibility results generalizing the observation that majority judgments can be logically inconsistent, a phenomenon which is referred to as the discursive dilemma. For an introductory overview of judgment aggregation theory, see papers in the Symposium on Judgment Aggregation in Journal of Economic Theory (C. List and B. Polak eds., 2010). I now selectively review the papers in judgment aggregation literature which consider strategic voting or take a truth-tracking approach in the sense that there is a true state of the world and voters share a common preference for reaching this true state in their collective decision. Dietrich and List (2007a) analyze strategic voting in judgment aggregation but in a setting where voters have private values instead of private information. See also related work by Nehring and Puppe $(2002,2007)$, Dokow and Falik (2012) and Dietrich (2016). Bovens and Rabinowicz (2006), List (2005) and Pivato (2013) are few contributions taking the truth-tracking approach, where they apply the Condorcet Jury Theorem to judgment aggregation without considering private information and strategic incentives. See List and Pettit (2011) for a philosophical analysis of the truth-tracking approach. The analysis of strategic voting under common preferences and private information is well-established in the literature on binary collective choice problems, which is about voting on one issue and started with the seminal works by Austen-Smith and Banks (1996) and Feddersen and Pesendorfer (1997). In this framework, voters share common preferences for reaching true decisions (with some degree of heterogeneity in preferences in the latter work) but they hold private information about what the truth might be. They show that it need not be rational for all voters to vote truthfully and taking this into account, they analyze asymptotic efficiency. Their observation gives rise to their analysis of voting rules which make truthful voting occur in equilibrium. 
The introduction of multiple issues to strategic voting and information aggregation problems is by Ahn and Oliveros $(2012,2013)$. While the first paper considers private values, the second assumes common values and is more relevant. In this paper, they compare the asymptotic efficiency of two different mechanisms: a joint trial where both issues are resolved by a majority vote among the group, and a severed trial where each issue is decided by a majority vote among a subgroup. They show that neither of these procedures is generally more efficient than the other one if the group is large enough. Bozbay, Dietrich and Peters (2014) aim to design voting rules that efficiently use all private information and lead to truthful voting. They find that in most cases, a quota rule, which decides each issue according to whether the proportion of 'yes' votes exceeds a particular threshold, can or should be used to guarantee efficient information aggregation. The present paper is an extension of their work to interconnected issues, and it turns out that when it comes to interconnected issues, there exists no quota rule which makes truthful voting efficient. De Clippel and Eliaz (2015) consider a setting where a group's optimal action (such as convicting or acquitting the defendant) depends on whether some premises are judged to be true by the group. They compare premise-based voting with conclusion-based voting. Under the former, a vote is taken on each issue, and the group action (conclusion) is determined by the outcome of voting which is decided by a super-majority rule. Under the latter, the group votes directly on which action to take, without forming a group decision on the issues. They show that premise-based voting is more efficient than conclusion-based voting, but that the difference vanishes asymptotically as the group size increases. All three aforementioned papers consider independent issues but the analysis is non-trivial given that preferences are non-separable and they establish links between issues. ${ }^{4}$ In the case of interconnected issues, there is an extra degree of non-separability, which follows from the fact that issues present a particular logical structure. This additional non-separability changes the structure of the voting rules that lead to efficient information aggregation.

\section{The Model}

\subsection{The judgment aggregation problem}

I consider a group of voters, labeled $i=1, \ldots, n$, where $n \geq 2$. This group needs a collective judgment on whether some proposition $p$ or its negation $\bar{p}$ is true, and whether some other proposition $q$ or its negation $\bar{q}$ is true. While doing so, voters know that

\footnotetext{
${ }^{4}$ For instance, the model in Ahn and Oliveros (2013) allows for a setting where accepting only a particular issue among two issues is the best (common) outcome while accepting both issues may be worse than rejecting both. Similarly, in Bozbay, Dietrich and Peters (2014) and De Clippel and Eliaz (2015), the optimal group action that is taken as a result of the vote may follow a standard which is not necessarily monotonic in the number of accepted issues. For instance, given the state of the world voters may be indifferent between accepting both issues and rejecting both issues while they prefer both of these outcomes to accepting only one issue (because these are the outcomes that lead to the correct group action).
} 
the combination $\{\bar{p}, \bar{q}\}$ is not possible. Hence, the interconnection between the two propositions is encoded by ' $\bar{p} \rightarrow q$ '. Any other type of interconnection between two issues (except bi-implication) is equivalent to this one up to isomorphism, so up to interchanging the roles of each proposition by its negation. Hence, studying this interconnection allows me to cover all possible interconnections between two propositions (except bi-implication, which is a trivial case). The three possible judgment sets are $\{p, q\},\{p, \bar{q}\},\{\bar{p}, q\}$, abbreviated by $p q, p \bar{q}$ and $\bar{p} q$, respectively. Similarly, $\{\bar{p}, \bar{q}\}$ is abbreviated by $\bar{p} \bar{q}$. Each voter votes for a judgment set in $\mathcal{J}=\{p q, p \bar{q}, \bar{p} q\}$. A collective decision in $\mathcal{J}$ is taken using a voting rule, defined as a function $f: \mathcal{J}^{n} \rightarrow \mathcal{J}$, which maps each voting profile $\mathbf{v}=\left(v_{1}, \ldots, v_{n}\right)$ to a decision $d=f(\mathbf{v})$.

\subsection{Truth-tracking preferences}

There is one 'correct' judgment set in $\mathcal{J}$, which I call the state (of the world) and denote by $s$. The state is unobservable by voters. Voters have identical preferences, represented by a common utility function $u: \mathcal{J} \times \mathcal{J} \rightarrow \mathbb{R}$ which maps any decisionstate pair $(d, s)$ to its utility $u(d, s)$. Since voters are truth-trackers, i.e., they want to reach the true state of the world, one should expect the utility to be high if the decision is correct. ${ }^{5}$ However, multiplicity of issues and the structure of the problem allow for different specifications. I mainly consider two kinds of preferences, namely, simple and consequentialist preferences. Under simple preferences, voters want to find out the state-matching decision. The utility function is given by

$$
u(d, s)= \begin{cases}1 & \text { if } d=s \text { (correct decision) } \\ 0 & \text { if } d \neq s \text { (incorrect decision) }\end{cases}
$$

To define consequentialist preferences, I assume that there are two possible consequences of voting, which represent group action. A consequence function Co maps the set $\mathcal{J}$ to a two-element set of possible consequences. Consider the example of the EU Commission, having to decide whether to supply additional funds to a candidate country. This depends on the collective judgments on two issues: $p$ : 'the country has implemented its benchmark political criteria' and $q$ : 'the country's economy is unstable', where the negation of the former implies the latter, $\bar{p} \rightarrow q$. If both issues are judged to be true, the consequence is to supply the funds, so $\operatorname{Co}(p q)=$ 'supply'. If only one of the issues is judged to be true, then the commission does not see the country as a good candidate for additional funds since they are either unnecessary or not deserved; so $\operatorname{Co}(p \bar{q})=\operatorname{Co}(\bar{p} q)=$ 'no supply'. ${ }^{6}$ Note that this consequence function with the property

$$
\operatorname{Co}(p q) \neq \operatorname{Co}(p \bar{q})=\operatorname{Co}(\bar{p} q)
$$

\footnotetext{
${ }^{5}$ Formally, under truth-tracking preferences, if $u(d, s)=\bar{u}$ when $d=s$, then $u\left(d^{\prime}, s\right) \leq \bar{u}$ for all $d^{\prime} \in \mathcal{J} \backslash\{d\}$ with strict inequality for at least one decision.

${ }^{6}$ The two possible consequences 'supply' and 'no supply' may also be represented by a conclusion proposition $c$ and its negation $\bar{c}$ respectively, as commonly done in judgment aggregation literature. Then, the consequence would be encoded in the biconditional $c \leftrightarrow(p \wedge q)$.
} 
is the only interesting consequence function up to isomorphism. That's because consequence functions which lead all decisions to the same consequence are degenerate and uninteresting. Moreover, if the consequence function depends only on the decision between $p$ and $\bar{p}$ (as in, $\operatorname{Co}(p q)=\mathrm{Co}(p \bar{q}) \neq \mathrm{Co}(\bar{p} q)$ ), or only on the decision between $q$ and $\bar{q}$ (as in, $\operatorname{Co}(p q)=\mathrm{Co}(\bar{p} q) \neq \operatorname{Co}(p \bar{q})$ ), then the problem reduces to making a judgment on a single proposition-negation pair which has already been studied in the literature on binary collective choice with common interests. So, studying the consequence function with the property (2) covers non-degenerate consequentialist preferences exhaustively. Throughout the paper, when considering consequentalist preferences, I will assume that the consequence function Co satisfies (2). Hence, I define the consequentialist utility function as

$$
u(d, s)= \begin{cases}1 & \text { if } \operatorname{Co}(d)=\operatorname{Co}(s) \text { (correct consequence) } \\ 0 & \text { if } \operatorname{Co}(d) \neq \operatorname{Co}(s) \text { (incorrect consequence) }\end{cases}
$$

where Co is any consequence function satisfying (2).

Simple preferences may describe an environment where judgments on issues do not necessarily lead to a consequence or action, or where voters want to reach the right action through correct reasons. Note that incorrect decisions may lead to correct consequences. On the other hand, under consequentialist preferences, voters want to reach the correct consequence no matter whether the underlying premises are correct or not.

\subsection{Private information and strategies}

Each voter has a type, which is an element of $\mathcal{T}=\{p q, p \bar{q}, \bar{p} q, \bar{p} \bar{q}\}$ and is denoted by $t$ generically. A voter's type represents evidence about whether $p$ is true and whether $q$ is true. For instance, the type $t=p \bar{q}$ represents evidence for $p$ and for $\bar{q}$, and the type $t=\bar{p} \bar{q}$ represents evidence for $\bar{p}$ and for $\bar{q}$, which overall is conflicting information ${ }^{7}$ since $\bar{p} \bar{q} \notin \mathcal{J}$. I write $\mathbf{t}=\left(t_{1}, \ldots, t_{n}\right) \in \mathcal{T}^{n}$ for a profile of voters' types.

Nature draws a state-types combination $(s, \mathbf{t})$ in $\mathcal{J} \times \mathcal{T}^{n}$ according to a probability measure denoted Pr. The prior probability of state $s \in \mathcal{J}$ is denoted

$$
\pi_{s}=\operatorname{Pr}(s)
$$

and is assumed to be in the interval $(0,1)$. If a proposition $r \in\{p, \bar{p}, q, \bar{q}\}$ represents (part of) voter $i$ 's type rather than (part of) the true state, I often write $r_{i}$ for $r$. I write $\operatorname{Pr}\left(p_{i} \mid p\right)$ for the probability that voter $i$ has evidence for $p$ given that $p$ is true. The probability of getting evidence for $r$ given that $r$ is true is denoted

$$
a_{r}=\operatorname{Pr}\left(r_{i} \mid r\right)
$$

and by assumption belongs to $(1 / 2,1)$ and does not depend on the voter $i$.

\footnotetext{
${ }^{7}$ Conflicting information occurs with positive probability in this model, given the standard independence assumptions on the distribution of types.
} 
I assume voters' types are independent given the state. Moreover, given the truth about $p$ (i.e., either $p$ or $\bar{p}$ ), a voter's evidence about $p$ (i.e., either $p_{i}$ or $\bar{p}_{i}$ ) is independent of the truth and the evidence about $q$; and similarly, given the truth about $q$, a voter's evidence about $q$ is independent of the truth and the evidence about $p$. The joint distribution of the state and the types is then given by

$$
\operatorname{Pr}(s, \mathbf{t})=\operatorname{Pr}(s) \times \prod_{i=1}^{n} \operatorname{Pr}\left(t_{i} \mid s\right) .
$$

Each voter votes for a judgment in $\mathcal{J}$ based on her type. A (voting) strategy is a function $\sigma: \mathcal{T} \rightarrow \mathcal{J}$, mapping each type $t \in \mathcal{T}$ to the type's vote $v=\sigma(t)$. I write $\boldsymbol{\sigma}=\left(\sigma_{1}, \ldots, \sigma_{n}\right)$ for a profile of voters' strategies. I say that a strategy profile $\boldsymbol{\sigma}$ is efficient if for every type profile $\mathbf{t}$, the resulting decision $d=f\left(\sigma_{1}\left(t_{1}\right), \ldots, \sigma_{n}\left(t_{n}\right)\right)$ is efficient, i.e., has maximal expected utility conditional on full information $\mathbf{t}$. This means the collective decision is no worse than a decision of a social planner who has full information.

Throughout the paper, I refer to ' $n, \pi_{s}$ for $s \in \mathcal{J}, a_{r}$ for $r \in\{p, \bar{p}, q, \bar{q}\}$ ' as model parameters, or simply, parameters. To avoid distraction by special cases, I make two assumptions on the combinations of these parameters:

Non-degeneracy: I exclude the combinations of the parameters where some decision in $\mathcal{J}$ is not efficient for any type profile.

No efficiency ties: I exclude efficiency ties, i.e., those special parameter combinations such that some type profile $\mathbf{t}$ leads to different efficient decisions except in the case that, for a given state, these decisions have the same utility independently of the model parameters and realization of types. Under simple preferences, I exclude those combinations of the parameters such that some type profile leads to multiple efficient decisions. Under consequentialist preferences, this amounts to excluding those combinations of the parameters such that some type profile leads to multiple efficient decisions with different consequences.

By the former assumption, I rule out those degenerate situations where no individual would ever prefer a certain state irrespective of the profile of signals. By the latter assumption, a voter is never indifferent between two decisions except in the case that these decisions lead to the same utility regardless of the combination of model parameters, such as decisions with the same consequence under consequentialist preferences. These assumptions or their analogues are standard in the literature on binary collective choice with common interests and shared by several works. 


\section{A general (im)possibility}

\subsection{Setting the stage}

My aim, besides efficiency, is to obtain simple-minded, truthful voting behavior in equilibrium. This type of voting behavior has been represented by the notions of informative strategy or sincere strategy in the strategic voting and binary collective choice literature. Informative strategy generally means revealing the type in the vote, without further calculations. Sincere strategy, on the other hand, is usually defined as voting as if one's own vote alone determined the outcome. Hence, a voter adopting the sincere strategy votes for the decision that maximizes her expected payoff given her type alone. I mean informative voting by truthful behavior in this paper. My objective is to design the voting rule in such a way that the rule will lead to efficient decisions on the basis of informative votes. Reaching efficient decisions is the most important goal here, since an efficient decision maximizes the expected utility of every voter as preferences are common. Ideally, this rule should also lead to simpleminded and truthful voting behavior; in other words, informative voting. Apart from not requiring much sophistication from a voter, what makes informative strategy desirable? In the model presented here (and in binary collective choice literature as well as its multi-issue extension), a social planner who has all the available information knows exactly which decision is the most likely to be true. In the absence of such prior knowledge, informative strategy is what reveals the valuable information about the true state. In a setting where votes reveal nothing about the private information, a social planner (or a mechanism designer) who cannot observe the types might be clueless about the efficient decision. Thus, it is desirable for the designer that voters are incentivized to vote informatively. So, as a second goal, informative voting should constitute an equilibrium, i.e., the profile of informative strategies must be a Nash equilibrium of the corresponding Bayesian game. My objective is reduced to finding out when - under which voting rule - informative voting is efficient as efficiency is the best outcome for every voter. This is an implication of a well-known result, Theorem 1 by McLennan (1998), which states that for any voting rule, an efficient strategy profile is an equilibrium. Hence, as long as I have a rule that renders informative voting efficient, both goals are reached: voters are incentivized to vote informatively and the voting rule aggregates these votes into the efficient decision. When I say a voting rule makes informative voting efficient, I mean, for every realization of types, the voting rule leads to the expected utility maximizing decision on the basis of informative votes. This voting rule should be designed so as to help finding the 'correct' alternative by making optimal use of all the private information scattered across the voters.

The challenge here is to define an informative strategy. As one type $-\bar{p} \bar{q}-$ cannot be revealed directly in the vote, I cannot use the standard definition where type matches the vote. Consequently, I consider three natural extensions of the standard notion of informative strategy. It turns out that whichever definition I adhere to, it is not possible to find a voting rule which works under any combination of the parameters of 
the model. Only under very strong conditions - and depending on the specific utility function - one might have a voting rule which leads to efficient outcomes on the basis of informative votes. These (partial) impossibilities are results of the inability to fully reveal private information in the votes, due to the possibility of conflicting information. In Section 4, I introduce the possibility of abstention which solves the former issue and makes efficient information aggregation a possibility. In Section 5, I rule out conflicting information by assuming jointly distributed types (instead of independently for each issue) at the cost of some symmetry assumptions. Once again, this leads to efficient information aggregation by a voting rule.

\subsection{General preferences}

To start with, I define an informative strategy. Generally, I call a strategy $\sigma$ of a voter informative if $\sigma(t)=t$ for all $t \in \mathcal{T} \backslash\{\bar{p} \bar{q}\}$. I will later add further restrictions on an informative strategy and obtain different types of informative strategies depending on how a strategy $\sigma$ maps the type $\bar{p} \bar{q}$. Before, I state the following theorem which shows that the current definition is too unrestrictive, and there is no rule which renders informative voting efficient without restrictions on the combination of parameters.

Theorem 1 Consider an arbitrary common utility function $u: \mathcal{J} \times \mathcal{J} \rightarrow \mathbb{R}$ that is truth-tracking. There is no voting rule for which informative voting is efficient.

Although the proof of this theorem is written for two issues only, the message of the theorem can be extended to the case of arbitrary number of issues some of which are interconnected. Consider a type that is inconsistent with any true state, and a type profile where every voter has this inconsistent type. According to the definition above, this type profile might lead to any voting profile under informative voting. So, the same decision(s) must be efficient for every possible type profile for efficient information aggregation to have a chance, and this is an uninteresting (and degenerate) case where private information plays no role whatsoever.

The theorem shows that the current definition of informative voting is not restrictive enough. Indeed, the type $\bar{p} \bar{q}$ still provides useful information about the true state of the world since the probability of getting correct information for each proposition is greater than $1 / 2$. So, it is natural that type $\bar{p} \bar{q}$ is not totally ignored. I now define three types of informative strategies which differ from each other in their approach to $t=\bar{p} \bar{q}$. All three share that when the type is different than $\bar{p} \bar{q}$, it is directly revealed in the vote as they are informative in the general sense defined above.

I say that an informative strategy $\sigma$ is of class 1 if $\sigma(\bar{p} \bar{q}) \in\{p \bar{q}, \bar{p} q\}$. Here, informativeness is open to behavior: one can choose between $p \bar{q}$ and $\bar{p} q$ under conflicting evidence. A similar definition which is not open to behavior is the following. An informative strategy $\sigma$ is of class 2 if $\sigma(\bar{p} \bar{q})=\operatorname{argmax}_{s \in\{p \bar{q}, \bar{p} q\}} \pi_{s}$. So, besides following the evidence as much as possible, an informative voter of class 2 also takes into account the prior probabilities and votes for $p \bar{q}$ or $\bar{p} q$ depending on which one 
has a higher prior probability. Finally, an informative strategy $\sigma$ is of class 3 if $\sigma(\bar{p} \bar{q})=\operatorname{argmax}_{d \in \mathcal{J}} E(u(d, S) \mid t=\bar{p} \bar{q})$, where $S$ denotes the random variable generating the state. Here, voters with conflicting types vote for the judgment set with the highest expected utility given the conflicting type. So, when the type is conflicting, one makes use of the private information by voting sincerely.

Note that in the case $\pi_{p \bar{q}}=\pi_{\bar{p} q}$, the informative strategy of class 2 is not decisive. Similarly, if there are multiple decisions that maximize $E(u(d, S) \mid t=\bar{p} \bar{q})$, the informative strategy of class 3 is not decisive. For the purpose of this section, I omit these cases, or assume that in such situations there is an agreement to follow a particular decision (for instance, if $\pi_{p \bar{q}}=\pi_{\bar{p} q}$, for every voter $\sigma(\bar{p} \bar{q})=p \bar{q}$ ). This is because the case where the informative strategy is open to behavior is already covered by the analysis of the informative strategy of class $1 .{ }^{8}$ What I aim by studying the other two kinds of strategies is to see whether restrictions on the votes of conflicting types help efficient information aggregation. The results, which I present below, show that informative voting of a certain class is efficient only under a very strong condition over the model parameters. So, even when informative strategy is fully restrictive and not open to behavior, it is not possible to obtain a general possibility. Before stating these conditions and the characterization result for each class of informative voting, I state these conditions, which I call joint efficiency 1 and joint efficiency 2. For the definition of the former, the following is needed: for all $M \subseteq\{1, \ldots, n\}$, let $\mathcal{T}^{n}(M)=\left\{\mathbf{t} \in \mathcal{T}^{n}:\left\{i: t_{i}=p q\right\}=M\right\}$.

Joint efficiency 1: For any $M \subseteq\{1, \ldots, n\}$, there exists a decision $d \in \mathcal{J}$ that is efficient for all $\mathbf{t} \in \mathcal{T}^{n}(M)$.

I denote by $\sigma^{\text {inf }}$ the unique informative strategy given class 2 or given class 3 . The corresponding informative strategy profile, where each voter votes informatively according to informative strategy of a certain class is denoted by $\boldsymbol{\sigma}^{\text {inf }}=\left(\sigma_{1}^{\text {inf }}, \ldots, \sigma_{n}^{\text {inf }}\right)$. For any vector $\mathbf{w} \in \mathcal{J}^{n}$, I define $\mathcal{T}^{n}(\mathbf{w})=\left\{\mathbf{t} \in \mathcal{T}^{n}: \boldsymbol{\sigma}^{\text {inf }}(\mathbf{t})=\mathbf{w}\right\}$.

Joint efficiency 2: For any $\mathbf{w} \in \mathcal{J}^{n}$, there exists a decision $d \in \mathcal{J}$ that is efficient for all $\mathbf{t} \in \mathcal{T}^{n}(\mathbf{w})$.

Theorem 2 Consider an arbitrary common utility function $u: \mathcal{J} \times \mathcal{J} \rightarrow \mathbb{R}$ that is truth-tracking. Assume informative voting of class 1 (class 2) [class 3]. There exists a voting rule for which informative voting is efficient if and only if joint efficiency 1 (joint efficiency 2) [joint efficiency 2] holds.

\footnotetext{
${ }^{8}$ As the first class of informative strategy is open to behavior, given a type profile $\mathbf{t}$, an informative strategy profile is not necessarily uniquely defined and the same type profile may produce different voting profiles. Informative voting of class 1 being efficient means that for any given type profile $\mathbf{t}$, every profile of corresponding informative strategies is efficient. A voting rule which makes some strategy profiles efficient and some not is not interesting since a designer never knows how these voters will vote. Under the class 2 or class 3 informative strategy, a type profile $\mathbf{t}$ is mapped to a unique voting profile.
} 
These conditions are clearly strong. Joint efficiency 1 requires that for all type profiles where the same group of voters have type $p q$, there must be a common efficient decision. Joint efficiency 2 requires that for all type profiles that produce the same voting profile, there must be a common efficient decision. ${ }^{9}$ Suppose the decision that maximizes $E(u(d, S) \mid t=\bar{p} \bar{q})$ is $\bar{p} q$ without loss of generality. Joint efficiency 2 imposes that the type profile $\mathbf{t}=(\bar{p} q, \ldots, \bar{p} q)$ and $\mathbf{t}^{\prime}=(\bar{p} \bar{q}, \ldots, \bar{p} \bar{q})$ should have a common efficient decision. While the former has full evidence for $q$ being true, the latter has the opposite, hence, full evidence for $q$ being not true. Yet, only when there is a common efficient decision for both, there is a chance for efficient information aggregation. The condition is strong, though implications may vary depending on the particular kind of truth-tracking preferences we consider. For instance, under a consequentialist framework, $q$ and $\bar{q}$ may have similar effects on the outcome, hence, on the utility. So, I now narrow the focus to the specific kind of preferences to see further implications of these conditions. I study simple and consequentialist preferences in turn in the following subsections, so that I can say more about the nature of voting rules making informative voting efficient whenever the relevant condition is satisfied.

\subsection{Simple preferences}

I start by addressing simple preferences, defined by (1), where correct decisions are preferred to incorrect ones. By focusing on simple preferences, can we say more than the (partial) existential claim of Theorem 2? The actual answer depends on which class of informative voting we focus on. For instance, it turns out that joint efficiency 1 never holds under simple preferences. Consequently, I have the following impossibility result.

Theorem 3 Under simple preferences, there exists no voting rule for which informative voting of class 1 is efficient.

It is possible that joint efficiency 2 holds under simple preferences, no matter if we focus on informative strategy of class 2 or 3 . Here is an example:

Example 1 Consider the following combination of parameters: $n=3, a_{p}=0.55$, $a_{q}=0.52, a_{\bar{p}}=0.55, a_{\bar{q}}=0.6, \pi_{p q}=0.3, \pi_{p \bar{q}}=0.5$ and $\pi_{\bar{p} q}=0.2$. If we focus on the informative voting of class $2, \sigma^{\text {inf }}(\bar{p} \bar{q})=p \bar{q}$ as $\pi_{p \bar{q}}>\pi_{\bar{p} q}$. If we instead focus on the informative voting of class 3 , once again $\sigma^{i n f}(\bar{p} \bar{q})=p \bar{q}$ since $d=p \bar{q}$ maximizes $E(u(d, S) \mid \bar{p} \bar{q})$. (The expressions that lead to these calculations are in Appendix.) So, every conclusion of this example applies to either of the two definitions. Joint efficiency 2 requires that the efficient decision is the same for all type profiles which lead to the same informative voting profile. The table below groups all such type profiles (up to permuting the order of the types). For each type profile in a group of

\footnotetext{
${ }^{9}$ It is clear that the interpretations of these two conditions are equivalent. One can alternatively define a unique condition which implies joint efficiency 1 under informative voting of class 1 and joint efficiency 2 under the other two classes. However, two separate conditions are preferable for simplicity of notation and for direct understanding of the conditions.
} 
type profiles leading to the same voting profile under informative voting, there is a common efficient decision.

Table 1: Under simple preferences, joint efficiency 2 for informative voting of type 2 holds for the following parameter combinations: $n=3, a_{p}=0.55, a_{q}=0.52, a_{\bar{p}}=0.55, a_{\bar{q}}=0.6, \pi_{p q}=0.3$, $\pi_{p \bar{q}}=0.5$ and $\pi_{\bar{p} q}=0.2$

\begin{tabular}{|c|c|c|c|}
\hline Group & Type profile $\mathbf{t}$ & $\begin{array}{c}\text { Voting profile } \\
\mathbf{v}=\boldsymbol{\sigma}^{i n f}(\mathbf{t})\end{array}$ & $\begin{array}{c}\text { Efficient decision for } \\
\mathbf{t}\end{array}$ \\
\hline \multirow{2}{*}{1} & $\begin{array}{l}(p \bar{q}, p \bar{q}, p \bar{q}) \\
(\bar{p} \bar{q}, \bar{p} \bar{q}, \bar{q}) \\
(p \bar{q}, p \bar{q}, \bar{p} \bar{q}) \\
(p \bar{q}, \bar{p} \bar{q}, \bar{p} \bar{q})\end{array}$ & $(p \bar{q}, p \bar{q}, p \bar{q})$ & $d^{*}=p \bar{q}$ \\
\hline 2 & $\begin{array}{l}(p q, p \bar{q}, p \bar{q}) \\
(p q, \bar{p} \bar{q}, \bar{p}) \\
(p q, p \bar{q}, \bar{q})\end{array}$ & $(p q, p \bar{q}, p \bar{q})$ & $d^{*}=p \bar{q}$ \\
\hline 3 & $\begin{array}{l}(\bar{p} q, p \bar{q}, p \bar{q}) \\
(\bar{p} q, \bar{p} \bar{q}, \bar{q})\end{array}$ & $(\bar{p} q, p \bar{q}, p \bar{q})$ & $d^{*}=p \bar{q}$ \\
\hline 4 & $\begin{array}{c}(p q, p \bar{q}, \bar{p} \bar{q}) \\
(p q, p q, p \bar{q})\end{array}$ & $(p q, p q, p \bar{q})$ & $d^{*}=p \bar{q}$ \\
\hline 5 & $\begin{array}{l}(\bar{p} q, \bar{p} q, p \bar{q}) \\
(\bar{p} q, \bar{p} q, \bar{p} \bar{q})\end{array}$ & $(\bar{p} q, \bar{p} q, p \bar{q})$ & $d^{*}=p \bar{q}$ \\
\hline 6 & $\begin{array}{l}(p q, p \bar{q}, p \bar{q}) \\
(p q, p \bar{q}, \bar{p} \bar{q})\end{array}$ & $(p q, p \bar{q}, p \bar{q})$ & $d^{*}=p \bar{q}$ \\
\hline
\end{tabular}

Table 1 does not give an exhaustive list of possible type profiles, but only groups of those which share the voting profile under informative voting. Note that the given parametrization satisfies the Non-degeneracy and No efficiency ties assumptions. For instance, $p q$ is the efficient decision for $\mathbf{t}=(\bar{p} q, p q, p q)$, and $\bar{p} q$ is the efficient decision for $\mathbf{t}=(\bar{p} q, \bar{p} q, \bar{p} q)$.

How often does joint efficiency 2 hold? Can we conclude that it always holds for a reasonable domain of combinations of model parameters? The answer is no, as small deviations from the above parameters lead to the failure of the condition. For instance, keeping all else the same, let $a_{q}=0.53$ instead of 0.52 . Joint efficiency 2 no longer holds. This is because in Group 5 above, the efficient decision for $\mathbf{t}=(\bar{p} q, \bar{p} q, \bar{p} \bar{q})$ is now $\bar{p} q$ while the efficient decision for $\mathbf{t}=(\bar{p} q, \bar{p} q, p \bar{q})$ is $p \bar{q}$. So, these two type profiles lead to the same voting profile under informative voting but they do not share a common efficient decision. Similar violations can be obtained by letting, for instance, $a_{p}=0.56$ or $a_{\bar{q}}=0.61$.

Remark 1 Under simple preferences, joint efficiency 2 holds for some combinations of parameters $n, a_{r}$ for $r \in\{p, \bar{p}, q, \bar{q}\}, \pi_{s}$ for $s \in \mathcal{J}$.

Regardless of whether the condition holds or not, one can still reason about most likely states given a voting profile. Consider a combination of model parameters and 
assume informative voting of some class (2 or 3 ) for which $\sigma^{\text {inf }}(\bar{p} \bar{q})=p \bar{q}$ without loss of generality. Let $\mathbf{t}$ be any type profile and $\mathbf{v}=\boldsymbol{\sigma}^{\text {inf }}(\mathbf{t})$. For each $r \in\{p, q\}$, I denote by $n_{r}^{\mathbf{t}}$ the number of types in $\mathbf{t}$ containing proposition $r$. Similarly, I define $n_{r}^{\mathbf{v}}$ for each $r \in\{p, q\}$ as the number of votes in $\mathbf{v}$ containing $r$. Given that $\bar{p} \bar{q}$ types in the type profile - whenever they exist - are replaced by $p \bar{q}$ in the voting profile, we must have $n_{p}^{\mathbf{v}} \geq n_{p}^{\mathbf{t}}$ and $n_{q}^{\mathbf{v}}=n_{q}^{\mathbf{t}}$. I denote by $\hat{\mathbf{t}}$ the type profile that is equivalent to $\mathbf{v}$, so, $\hat{\mathbf{t}}=\mathbf{v}$. What determines the efficient decision given a type profile is the number of occurrences of each proposition in the profile. An efficient decision for a type profile with $k$ number of evidence for $p$ and $l$ number of evidence for $q$ is given by $F(k, l)$. We then have the following:

- If $F\left(n_{p}^{\mathbf{v}}, n_{q}^{\mathbf{v}}\right)=p q$, then $F\left(n_{p}^{\mathbf{t}}, n_{q}^{\mathbf{t}}\right) \in\{p q, \bar{p} q\}$;

- If $F\left(n_{p}^{\mathbf{v}}, n_{q}^{\mathbf{v}}\right)=p \bar{q}$, then $F\left(n_{p}^{\mathbf{t}}, n_{q}^{\mathbf{t}}\right) \in\{p \bar{q}, \bar{p} q\}$;

- If $F\left(n_{p}^{\mathbf{v}}, n_{q}^{\mathbf{v}}\right)=\bar{p} q$, then $F\left(n_{p}^{\mathbf{t}}, n_{q}^{\mathbf{t}}\right)=\bar{p} q$.

Under a conveniently defined voting rule ${ }^{10}$, informative voting leads to the efficient decision with certainty in one case out of the three possible cases and rules out a certainly inefficient decision in two cases out of the three. An analogous analysis can be written for the case where $\sigma^{i n f}(\bar{p} \bar{q})=\bar{p} q$. For the case where $\sigma^{i n f}(\bar{p} \bar{q})=p q$ (which is only possible for informative strategy of class 3 ), the analysis changes slightly, since now neither the number of $p$ types nor the number of $q$ types can be learned from the voting profile. Yet, one can still derive similar conditions and focus on a class of voting rules which, at the least, rules out one inefficient decision for each case.

The example and the discussion that follows, shows the fragility of joint efficiency 2 , hence, the fragility of efficient information aggregation. The reasoning behind is the impossibility of revealing all available information. Before I propose ways to overcome this problem, I focus on the implications of joint efficiency on consequentialist preferences in the next section.

\subsection{Consequentialist preferences}

What are the implications of joint efficiency ( 1 and 2$)$ under consequentialist preferences? To start with, I ask the question whether the impossibility obtained for simple preferences with joint efficiency 1 persists under consequentialist preferences. I state the answer to this question after defining the following two coefficients.

$$
\begin{aligned}
& A=\pi_{p \bar{q}}\left(\frac{1-a_{\bar{q}}}{a_{q}}\right)^{n}+\pi_{\bar{p} q}\left(\frac{1-a_{\bar{p}}}{a_{p}}\right)^{n-1} \frac{a_{\bar{p}}}{1-a_{p}}, \\
& B=\pi_{p \bar{q}}\left(\frac{1-a_{\bar{q}}}{a_{q}}\right)^{n-1} \frac{a_{\bar{q}}}{1-a_{q}}+\pi_{\bar{p} q}\left(\frac{1-a_{\bar{p}}}{a_{p}}\right)^{n} .
\end{aligned}
$$

\footnotetext{
${ }^{10}$ This rule should naturally satisfy the following: For a voting profile $\mathbf{v} \in \mathcal{J}, f(\mathbf{v})=\bar{p} q$ if $F\left(n_{p}^{\mathbf{v}}, n_{q}^{\mathbf{v}}\right)=\bar{p} q ; f(\mathbf{v}) \in\{p q, \bar{p} q\}$ if $F\left(n_{p}^{\mathbf{v}}, n_{q}^{\mathbf{v}}\right)=p q ; f(\mathbf{v}) \in\{p \bar{q}, \bar{p} q\}$ if $F\left(n_{p}^{\mathbf{v}}, n_{q}^{\mathbf{v}}\right)=p \bar{q}$.
} 
Theorem 4 Under consequentialist preferences, there exists a voting rule for which informative voting of class 1 is efficient if and only if the decision $p q$ is efficient only for the unanimous type profile $\mathbf{t}=(p q, \ldots, p q)$ (which is the case if and only if $\left.A, B>\pi_{p q}\right)$.

The theorem states that informative voting of class 1 can be efficient under consequentialist preferences if $p q$ is the efficient decision only when there is perfect evidence for $p q$. This is what joint efficiency 1 reduces to under consequentialist preferences. To satisfy this condition, the prior probability of $p q$ should be sufficiently low. For instance, if $\pi_{p q}=\pi_{\bar{p} q}=0.3, \pi_{p \bar{q}}=0.4, a_{p}=a_{q}=a_{\bar{p}}=a_{\bar{q}}=0.6$ and $n=3$, such a voting rule exists whereas no voting rule makes informative voting efficient if instead $\pi_{p q}=0.35, \pi_{\bar{p} q}=0.25$ and $\pi_{p \bar{q}}=0.4$.

The judgment sets $p \bar{q}$ and $\bar{p} q$ both lead to the same consequence according to (2), so the same utility by (3). Consider a type profile $\mathbf{t}$ with $t_{i}=p \bar{q}$ for all $i \in\{1, \ldots, n\}$ and a type profile $\mathbf{t}^{\prime}$ with $t_{i}^{\prime}=\bar{p} q$ for all $i \in\{1, \ldots, n\}$. Joint efficiency 1 , the existence condition for efficient information aggregation, would require that there is a common efficient decision for both $\mathbf{t}$ and $\mathbf{t}^{\prime}$. This is of course not possible under simple preferences (by Non-degeneracy), while it follows under consequentialist preferences. Hence, the possibility in Theorem 4 relies on the fact that voters are indifferent between the two judgment sets $p \bar{q}$ and $\bar{p} q$ given the assumption that $\operatorname{Co}(p q) \neq \operatorname{Co}(p \bar{q})=\operatorname{Co}(\bar{p} q)$ and that preferences are defined by (3).

I now present a simple characterization of voting rules which make informative voting efficient under consequentialist preferences when the condition is satisfied.

Proposition 1 Assume consequentialist preferences and joint efficiency 1. A voting rule $f: \mathcal{J}^{n} \rightarrow \mathcal{J}$ makes informative voting of class 1 efficient if and only if for every voting profile $\mathbf{v} \in \mathcal{J}^{n}, f(\mathbf{v})=p q$ if $\mathbf{v}=(p q, \ldots, p q)$ and $f(\mathbf{v}) \in\{p \bar{q}, \bar{p} q\}$ otherwise.

Proposition 1 describes a class of voting rules which accept $p q$ only when there is unanimous agreement about both issues being true. Some of these rules characterized by Proposition 1 satisfy some natural properties (and others do not). In Appendix A.1, I introduce some desirable properties of a voting rule to characterize a subclass of voting rules given in Proposition 1. The part shows that one can still attain desirable properties of a voting rule without compromising on efficient information aggregation.

What about informative voting of class 2 and class 3 ? How likely is it that joint efficiency 2 holds? The indifference of voters between the two judgment sets $p \bar{q}$ and $\bar{p} q$ under consequentialist preferences naturally allows to obtain a larger domain of parameters where the condition holds compared to under simple preferences. Let me illustrate this with an example similar to Example 1 above.

Example 2 Consider the following combination of parameters: $n=3, a_{p}=0.55$, $a_{q}=0.52, a_{\bar{p}}=0.55, a_{\bar{q}}=0.6, \pi_{p q}=0.33, \pi_{p \bar{q}}=0.47$ and $\pi_{\bar{p} q}=0.2$. (These parameters differ from those of Example 1 only by the slight modifications in prior probabilities. This modification was necessary to satisfy the Non-degeneracy assump- 
tion.) Under informative voting of type $2, \sigma^{i n f}(\bar{p} \bar{q})=p \bar{q}$ while under informative voting of type 3, a sincere voter would be indifferent between $p \bar{q}$ and $\bar{p} q$ given the assumption $\operatorname{Co}(p \bar{q})=\operatorname{Co}(\bar{p} q)$. Let me assume that in that case, an informative voter votes for the judgment set with a higher prior probability; in this case, for $p \bar{q}$. This parametrization gives the same table as Table 1, but now, $\bar{p} q$ should be added to the last column as the other efficient decision. So, joint efficiency 2 is satisfied. Unlike under simple preferences, keeping all else fixed and increasing $a_{q}$ or $a_{p}$, joint efficiency 2 continues to hold.

The impossibility results of this section are mainly driven by the fact that some voters receive information that cannot be revealed fully due to the incompatibility with the possible states. I remove this incompatibility by assuming jointly distributed private information for each issue in Section 5 (at the cost of some symmetry assumptions) and find that efficient information aggregation is always possible. Nonetheless, conflicting information is expected in the case of multiple interconnected issues, especially when types are distributed separately for issues than jointly. In next section, I propose abstention as a way to escape from impossibility of efficient information aggregation even when conflicting information is present.

\section{Abstention}

A voter with a conflicting type knows that she is 'ill-informed' about the true state. As voters share a common goal, she may prefer to abstain in the hope that better informed voters will determine the decision. In the context of Feddersen and Pesendorfer (1996) where a fraction of voters has state-dependent common preferences, a less informed voter prefers to abstain even when there is no cost to voting. Here, if conflicting types abstain while other types vote informatively, the problem of correctly revealing information is solved. Then, all is left to show is that such strategy profile is an equilibrium. As long as the resulting strategy profile is efficient, it is an equilibrium by the previously mentioned result of McLennan (1998). So, my aim is, once again, to design voting rules which make such strategy profile efficient.

I use the same notation and model as before with the exception of the definition of a voting strategy. Let $\mathcal{J}_{\phi}=\{p q, p \bar{q}, \bar{p} q, \phi\}$ be the set of possible votes where $\phi$ indicates abstention. For this section, I define a (voting) strategy as a function $\sigma: \mathcal{T} \rightarrow \mathcal{J}_{\phi}$. As before, I write $\boldsymbol{\sigma}=\left(\sigma_{1}, \ldots, \sigma_{n}\right)$ for a profile of voters' strategies. In this section, a voting rule $f: \mathcal{J}_{\phi}^{n} \rightarrow \mathcal{J}$ maps each voting profile $\mathbf{v}=\left(v_{1}, \ldots, v_{n}\right)$ to a decision $d=f(\mathbf{v})$.

The informative strategy with abstention is the voting strategy $\sigma$ with $\sigma(t)=t$ for all $t \in \mathcal{T} \backslash\{\bar{p} \bar{q}\}$ and $\sigma(\bar{p} \bar{q})=\phi$.

Theorem 5 Consider an arbitrary common utility function $u: \mathcal{J} \times \mathcal{J} \rightarrow \mathbb{R}$ that is truth-tracking. There always exists a voting rule which makes informative voting with abstention efficient (hence, an equilibrium). 
This theorem simply states that one can always find a mechanism which lead to efficient decisions when every voter votes according to the informative strategy with abstention. If a (virtual) social planner implements this rule, no voter with the inconsistent type has an incentive to vote and no other voter has an incentive to abstain instead. To understand the properties of the rule making informative voting with abstention efficient, one needs to focus on the exact kind of preferences. Among the various voting rules that are studied in the literature, quota rules are particularly common. Formally, a quota rule is given by two thresholds $m_{p}, m_{q} \in\{0,1, \ldots, n+1\}$, and for each voting profile it accepts $p[q]$ if and only if at least $m_{p}\left[m_{q}\right]$ voters accept it in the profile. However, as $\bar{p} \bar{q}$ cannot be chosen, not every combination of thresholds would lead to a well-defined voting rule. ${ }^{11}$ I focus on a class of voting rules, called quota rules with exception, which behave as a quota rule when at least one of the issues is accepted by a number of people equal to or more than the acceptance threshold. When neither issue reaches the acceptance threshold, as $\bar{p} \bar{q}$ is not an admissible state, the rule diverges from a quota rule and returns a decision according to an exception rule. The structure of the exception rule may depend on the specific utility function in use.

I analyze simple and consequentialist preferences in turn.

\subsection{Simple Preferences}

In the next result, I show that the quota rule with exception with particular thresholds and an exception rule is the only rule one can use to make informative voting with abstention efficient. To state the result, I start by introducing some notation. Let $\beta_{r}(k)=\left(\frac{1-a_{\bar{r}}}{a_{r}}\right)^{k}\left(\frac{a_{\bar{r}}}{1-a_{r}}\right)^{n-k}$ for each $r \in\{p, q\}$ and $k \in\{0, \ldots, n\}$. I consider a quota rule with exception $f$ with thresholds $m_{p}, m_{q}$ and with the exception rule given as

$$
n_{r}^{\mathbf{v}}<m_{r} \text { for both } r \in\{p, q\} \text { and } \frac{\pi_{p \bar{q}}}{\pi_{\bar{p} q}}>[<] \frac{\beta_{p}\left(n_{p}^{\mathbf{v}}\right)}{\beta_{q}\left(n_{q}^{\mathbf{v}}\right)} \Rightarrow f(\mathbf{v})=p \bar{q}[\bar{p} q] .
$$

I define two coefficients: ${ }^{12}$

$$
\begin{aligned}
& k_{p}=\min \left\{k \in\{0,1, \ldots, n\}: \frac{\pi_{p q}}{\pi_{\bar{p} q}}>\beta_{p}(k)\right\}, \\
& k_{q}=\min \left\{k \in\{0,1, \ldots, n\}: \frac{\pi_{p q}}{\pi_{p \bar{q}}}>\beta_{q}(k)\right\} .
\end{aligned}
$$

\footnotetext{
${ }^{11}$ For a quota rule to be well-defined in this framework, one must have $m_{p}+m_{q} \leq n+1$. The additional requirement of $m_{p}+m_{q} \leq n+1$ is for leaving out $\bar{p} \bar{q}$ from possible outcomes. This requirement follows from Theorem 2 (c) in Dietrich and List (2007b). Limiting attention to these rules would not necessarily lead to efficiency. As the focus here is on efficient information aggregation instead of procedural requirements, one has to depart from quota rules.

${ }^{12}$ The minimum defining $k_{p}$ or $k_{q}$ is taken over a nonempty set since emptiness is impossible under simple preferences. This follows from the Non-degeneracy assumption on the model parameters.
} 
These coefficients have an interpretation: as can be proved, for $p[q]$ to be more probably true than false given all information and all else equal, at least $k_{p}\left[k_{q}\right]$ individuals need to receive evidence for $p[q]$, i.e., need to have a type containing $p$ $[q]$. Note that for each $r \in\{p, q\}, \beta_{r}(k)$ is a decreasing function of $k$. So, $\beta_{r}$ will decrease when the number of types supporting $r$ increases, and the inequality will be more likely to hold; or in other words, $r$ will be more likely to be true than false.

I am now ready to state the result.

Theorem 6 Assume simple preferences. Informative voting with abstention is efficient if and only if the voting rule is the quota rule with exception with thresholds $k_{p}$, $k_{q}$ and the exception rule given by (4).

This result shows that the quota rule with exception with thresholds $k_{p}$ and $k_{q}$ and the exception rule (4) is the only rule one may use in view of making informative voting with abstention efficient.

\subsection{Consequentialist Preferences}

I now turn to consequentialist preferences when abstention is a possibility. I first propose a simple characterization result for voting rules which make informative voting with abstention efficient. As expected, the class of voting rules in this result is different than the rule characterized in Theorem 6 .

For $k, l \in\{0,1, \ldots, n\}, I$ define the coefficient

$$
\gamma(k, l)=\frac{\pi_{p q} a_{p}^{k}\left(1-a_{p}\right)^{n-k} a_{q}^{l}\left(1-a_{q}\right)^{n-l}}{\sum_{s \in \mathcal{J}} \pi_{s} a_{s_{p}}^{k}\left(1-a_{s_{p}}\right)^{n-k} a_{s_{q}}^{l}\left(1-a_{s_{q}}\right)^{n-l}}
$$

where $s_{r} \in\{r, \bar{r}\}$ is the value of proposition $r \in\{p, q\}$ in state $s$. The coefficient $\gamma(k, l)$ has a natural interpretation. It is the probability that the state is $p q$ conditional on having $k$ times evidence for (and $n-k$ times evidence against) $p$ and $l$ times evidence for (and $n-l$ times evidence against) $q$. So, $\gamma(k, l)=\operatorname{Pr}(p q \mid \mathbf{t})$ for some (hence, any) type profile $\mathbf{t} \in \mathcal{T}^{n}$ containing $p$ exactly $k$ times and $q$ exactly $l$ times. As $\operatorname{Co}(p q) \neq \operatorname{Co}(p \bar{q})=\operatorname{Co}(\bar{p} q)$, given a type profile $\mathbf{t}, E(u(d, S) \mid \mathbf{t})=\operatorname{Pr}(p \bar{q} \mid \mathbf{t})+\operatorname{Pr}(\bar{p} q \mid \mathbf{t})$ for $d \in\{p \bar{q}, \bar{p} q\}$. Hence, given a type profile $\mathbf{t}$ containing $p$ exactly $k$ times and $q$ exactly $l$ times, the only efficient decision is $p q$ if $\gamma(k, l)>1 / 2$. Otherwise, the two other decisions are both efficient. This implies the following simple characterization result.

Proposition 2 Assume consequentialist preferences. A voting rule $f$ makes informative voting with abstention efficient if and only if for every voting profile $\mathbf{v} \in \mathcal{J}_{\phi}^{n}$ the decision $f(\mathbf{v})$ is $p q$ if $\gamma\left(n_{p}^{\mathbf{v}}, n_{q}^{\mathbf{v}}\right)>1 / 2$ and in $\{p \bar{q}, \bar{p} q\}$ otherwise.

I do not provide a formal proof for this proposition as the claim can easily be shown by elaborating the argument above. For a given type profile $\mathbf{t}$, whenever $\gamma\left(n_{p}^{\mathbf{t}}, n_{q}^{\mathbf{t}}\right)>1 / 2$, $E(u(p q, S) \mid \mathbf{t})$ is maximal, hence, $p q$ is the efficient decision. When every voter follows informative strategy with abstention, in the resulting voting profile $\mathbf{v}$, one must have 
$n_{r}^{\mathbf{v}}=n_{r}^{\mathbf{t}}$ for each $r \in\{p, q\}$. As long as the rule selects $p q$ only when $\gamma\left(n_{p}^{\mathbf{v}}, n_{q}^{\mathbf{v}}\right)>1 / 2$, the efficiency is guaranteed.

What about quota rules with exception? Can one use this natural class of voting rules to guarantee efficient information aggregation? Unlike when preferences are simple, for some combinations of the model parameters, no quota rule with exception makes informative voting with abstention efficient. I characterize those combinations for which this class of rules is a possibility. I begin by introducing two coefficients.

$$
\begin{aligned}
& l_{p}=\min \{k \in\{0, \ldots, n\}: \gamma(k, n)>1 / 2\} \\
& l_{q}=\min \{k \in\{0, \ldots, n\}: \gamma(n, k)>1 / 2\} .
\end{aligned}
$$

The coefficient $l_{p}\left(l_{q}\right)$ has a simple interpretation. Given that all voters hold $q(p)$ type, it is the number of voters with evidence for $p(q)$ it minimally takes for the decision $p q$ to be the efficient decision. By Non-degeneracy, $p q$ must be efficient for at least one type profile; so, these two minima are taken over non-empty sets of values of $k$.

Theorem 7 Assume consequentialist preferences. There exists a quota rule with exception making informative voting with abstention efficient if and only if $\gamma\left(l_{p}, l_{q}\right)>$ $1 / 2$. In this case, this quota rule with exception has the thresholds $l_{p}$ and $l_{q}$ and an exception rule satisfying

$$
n_{r}^{\mathbf{v}}<l_{r} \text { for each } r \in\{p, q\} \Longrightarrow f(\mathbf{v}) \in\{p \bar{q}, \bar{p} q\} .
$$

As Theorem 7 shows, aiming for a quota rule with exception is not feasible for some combinations of values of the model parameters. For instance, let $n=3, \pi_{p q}=0.5$, $\pi_{p \bar{q}}=0.2, \pi_{\bar{p} q}=0.3, a_{p}=a_{\bar{p}}=0.62, a_{q}=a_{\bar{q}}=0.6$. For this combination, $l_{p}=2$ and $l_{q}=1$, however, $\gamma(2,1)<1 / 2$. On the other hand, if I let $a_{\bar{p}}=0.6$ keeping all else the same, $\gamma(2,1)>1 / 2$ and the quota rule with exception with thresholds $l_{p}=2$ and $l_{q}=1$ makes informative voting efficient.

While Proposition 2 states a simple characterization of the type of voting rules making informative voting with abstention efficient, it is generally possible to say more. Among the voting rules described by Proposition 2, I illustrate two of them. The first one takes the form

$$
f(\mathbf{v})= \begin{cases}p q & \text { if } \gamma\left(n_{p}^{\mathbf{v}}, n_{q}^{\mathbf{v}}\right)>1 / 2 \\ \bar{p} q & \text { if } \gamma\left(n_{p}^{\mathbf{v}}, n_{q}^{\mathbf{v}}\right) \leq 1 / 2\end{cases}
$$

for all $\mathbf{v} \in \mathcal{J}_{\phi}^{n}$. I can analogously define a voting rule where the resulting decision is $p \bar{q}$ instead of $\bar{p} q$ when $\gamma\left(n_{p}^{\mathbf{v}}, n_{q}^{\mathbf{v}}\right) \leq 1 / 2$. Following Proposition 2, (11) clearly renders informative voting with abstention efficient. Next, I define another interesting rule which satisfies some desirable properties and always makes informative voting with 
abstention efficient under some restrictions. Let

$$
f(\mathbf{v})= \begin{cases}p q & \text { if } \gamma\left(n_{p}^{\mathbf{v}}, n_{q}^{\mathbf{v}}\right)>1 / 2, \text { or } \\ & \text { if } n_{p}^{\mathbf{v}} \geq m_{p} \text { and } n_{q}^{\mathbf{v}} \geq m_{q} \\ p \bar{q} & \text { if } \gamma\left(n_{p}^{\mathbf{v}}, n_{q}^{\mathbf{v}}\right) \leq 1 / 2, n_{p}^{\mathbf{v}} \geq m_{p} \text { and } n_{q}^{\mathbf{v}}<m_{q}, \text { or } \\ & \text { if } \gamma\left(n_{p}^{\mathbf{v}}, n_{q}^{\mathbf{v}}\right) \leq 1 / 2, n_{p}^{\mathbf{v}}<m_{p}, n_{q}^{\mathbf{v}}<m_{q} \text { and } n_{p}^{\mathbf{v}}>n_{q}^{\mathbf{v}} \\ \bar{p} q \quad \text { if } \gamma\left(n_{p}^{\mathbf{v}}, n_{q}^{\mathbf{v}}\right) \leq 1 / 2, n_{p}^{\mathbf{v}}<m_{p} \text { and } n_{q}^{\mathbf{v}} \geq m_{q}, \text { or } \\ & \text { if } \gamma\left(n_{p}^{\mathbf{v}}, n_{q}^{\mathbf{v}}\right) \leq 1 / 2, n_{p}^{\mathbf{v}}<m_{p}, n_{q}^{\mathbf{v}}<m_{q} \text { and } n_{p}^{\mathbf{v}} \leq n_{q}^{\mathbf{v}}\end{cases}
$$

for all $\mathbf{v} \in \mathcal{J}_{\phi}^{n}$ and some thresholds $m_{p}, m_{q} \in\{0, \ldots, n\}$. Let $l_{p}^{\prime}=\min \{k \in\{0, \ldots, n\}$ : $\left.\gamma\left(k, l_{q}\right)>1 / 2\right\}$ and $l_{q}^{\prime}=\min \left\{k \in\{0, \ldots, n\}: \gamma\left(l_{p}, k\right)>1 / 2\right\}$. It is easy to see that if thresholds $m_{p}, m_{q}$ in (12) are chosen such that $m_{p}=l_{p}^{\prime}$ and $m_{q}=l_{q}^{\prime}$, informative voting with abstention is efficient with (12). This rule is special in the sense that whenever $\gamma\left(l_{p}, l_{q}\right)>1 / 2$, we necessarily have $m_{p}=l_{p}=l_{p}^{\prime}$ and $m_{q}=l_{q}=l_{q}^{\prime}$; so, it is contained by the set of the quota rules with exception described in Theorem 7 . Note that under this choice of thresholds, $\gamma\left(m_{p}, m_{q}\right)>1 / 2$ by the definitions of $l_{p}^{\prime}$ and $l_{q}^{\prime}$ and by $\gamma$ being an increasing function in both arguments.

The voting rule (11) is illustrated in Figure 1a). The voting rule (12) is illustrated in Figure 1b)\&c) for different choice of thresholds. In Figure 1b), thresholds $m_{p}, m_{q}$ are chosen such that $m_{q}>m_{p}$ and $\gamma\left(m_{p}, m_{q}\right)<1 / 2$. In Figure 1c), thresholds $m_{p}, m_{q}$ are chosen such that $m_{p}=l_{p}^{\prime}$ and $m_{q}=l_{q}^{\prime}$ which imply $\gamma\left(m_{p}, m_{q}\right)>1 / 2$. While the rules described in Figure 1a) and Figure 1c) make informative voting efficient, the one described in Figure 1b) does not.

a)

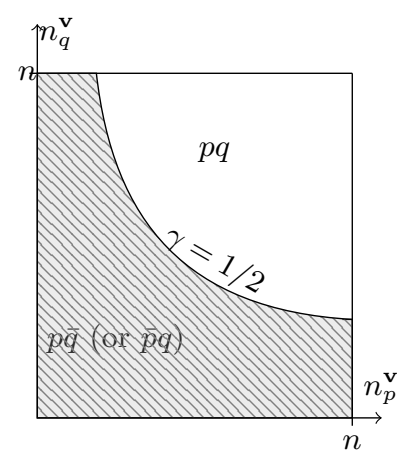

b)

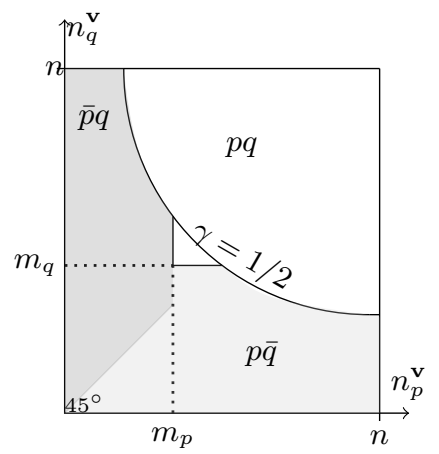

c)

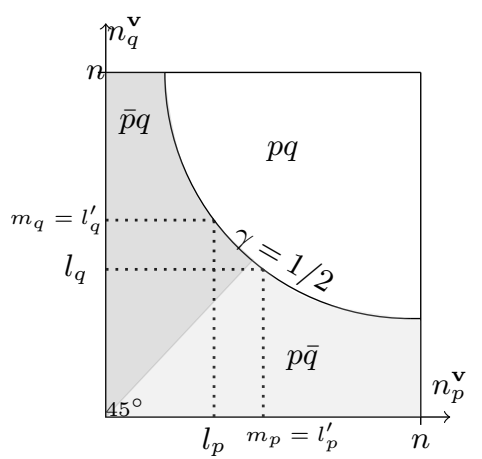

Figure 1: Illustration of the decision as a function of the number of votes for $p\left(n_{p}^{\mathbf{v}}\right)$ and $q\left(n_{q}^{\mathbf{v}}\right)$. Part a) illustrates (11) (or its analogue), Part b) illustrates (12) for $m_{p}, m_{q}$ such that $m_{q}>m_{p}$ and $\gamma\left(m_{p}, m_{q}\right)<1 / 2$, and Part c) illustrates (12) for $m_{p}=l_{p}^{\prime}$ and $m_{q}=l_{q}^{\prime}$. In Part a) the white area shows the voting profiles which lead to $p q$ and the shaded area shows the voting profiles which lead to $p \bar{q}$ (or $\bar{p} q$ ). In Part b) and in Part c), the light gray (dark gray) [white] area illustrates the voting profiles which lead to $p \bar{q}(\bar{p} q)[p q]$ under $(12)$. 


\section{The case of jointly distributed private information}

I now consider a setting where I introduce new assumptions on the distribution of types, while the judgment aggregation problem, preferences and prior probabilities are defined as in Section 2. A voter's type takes the form of an element of $\mathcal{J}$, generically denoted by $t$ as before. Following the previous notation for simplicity, I write $\mathbf{t}=\left(t_{1}, \ldots, t_{n}\right)$ for a profile of voters' types. Nature draws a state-types combination $(s, \mathbf{t}) \in \mathcal{J}^{n+1}$ according to a probability measure denoted Pr. I assume that the parts of the type for $p$ and $q$ are distributed jointly given the state, and the probability of getting evidence for $s$ given that $s$ is true is denoted by $a=\operatorname{Pr}(t=s \mid s)$ for every $s \in \mathcal{J}$, belongs to $(1 / 2,1)$, and does not depend on the voter $i$. The lower bound of $1 / 2$ reflects the (standard) idea that information is more reliable than a fair coin. Consequently, $\operatorname{Pr}(t \neq s \mid s)=1-a<1 / 2$. In this setting, the conflicting type $\bar{p} \bar{q}$ can no longer be observed by voters. To obtain a tractable model and derive interpretable properties, I assume symmetry concerning the likelihood of receiving correct information. So, the probability of receiving correct information is the same across states. Each voter submits a vote in $\mathcal{J}$ based on her type. A (voting) strategy is a function $\sigma: \mathcal{J} \rightarrow \mathcal{J}$, mapping each type $t \in \mathcal{J}$ to the type's vote $v=\sigma(t)$. As before, I write $\boldsymbol{\sigma}=\left(\sigma_{1}, \ldots, \sigma_{n}\right)$ for a profile of voters' strategies. A strategy $\sigma$ of a voter is informative if $\sigma(t)=t$ for all types $t$. Just as in the benchmark model, one defines the notions of an efficient decision given a type profile, an efficient strategy profile, and an equilibrium strategy profile.

Theorem 8 Consider an arbitrary common utility function $u: \mathcal{J} \times \mathcal{J} \rightarrow \mathbb{R}$ that is truth-tracking. There always exists a voting rule which makes informative voting efficient.

I first focus on simple preferences. In what follows below, the term $\frac{a}{1-a}(>1)$ plays an important role, which is the likelihood ratio of correct information to incorrect information given any state. It turns out that there is a uniquely defined voting rule that should be imposed to guarantee efficient information aggregation under simple preferences. Before I define this rule and state the theorem, let me introduce two functions which will be useful in what follows for a given $n$. For each $k, l \in\{0,1, \ldots, n\}$, let $\alpha(k, l)=\left(\frac{a}{1-a}\right)^{2 n-2 k-l}$, and $\beta(k, l)=\left(\frac{a}{1-a}\right)^{k-l}$. For any voting profile $\mathbf{v} \in \mathcal{J}$, let

$$
f(\mathbf{v})= \begin{cases}p q & \text { if } \frac{\pi_{p q}}{\pi_{\bar{p} q}}>\alpha\left(n_{p}^{\mathbf{v}}, n_{q}^{\mathbf{v}}\right) \text { and } \frac{\pi_{p q}}{\pi_{n_{\bar{q}}}}>\alpha\left(n_{q}^{\mathbf{v}}, n_{p}^{\mathbf{v}}\right) \\ \bar{p} q & \text { if } \frac{\pi_{p_{q}}}{\pi_{\bar{p} q}}<\alpha\left(n_{p}^{\mathbf{v}}, n_{q}^{\mathbf{v}}\right) \text { and } \frac{\pi_{\bar{p} q}}{\pi_{p \bar{q}}}>\beta\left(n_{p}^{\mathbf{v}}, n_{q}^{\mathbf{v}}\right) \\ p \bar{q} & \text { otherwise. }\end{cases}
$$

Theorem 9 Assume simple preferences. Informative voting is efficient if and only if the voting rule is given by (13).

Unlike in the setting where signals for each proposition are distributed independently and abstention is possible, a quota rule with exception does not lead to efficient information aggregation in this setting. The reasoning is simple. Private information 
containing, say, $p$ does not necessarily carry evidence for both $p q$ and $p \bar{q}$ as before, but all depends on whether the type also contains $q$ or $\bar{q}$. Hence, the effect of $p$ evidence is no longer balanced out when one compares the likelihood of $p q$ and $p \bar{q}$. What about consequentialist preferences? I start by a simple characterization, and then move on to the more specific case of quota rules with exception. The message of the proposition is straightforward, so I do not provide a formal proof.

Proposition 3 Assume consequentialist preferences. A voting rule $f$ makes informative voting efficient if and only if for every voting profile $\mathbf{v} \in \mathcal{J}^{n}$ the decision $f(\mathbf{v})$ is $p q$ if $\pi_{p q}>\pi_{\bar{p} q} \alpha\left(n_{p}^{\mathbf{v}}, n_{q}^{\mathbf{v}}\right)+\pi_{p \bar{q}} \alpha\left(n_{q}^{\mathbf{v}}, n_{p}^{\mathbf{v}}\right)$ and in $\{p \bar{q}, \bar{p} q\}$ otherwise.

I define two coefficients to state the next result:

$$
\begin{aligned}
& h_{p}=\min \left\{k \in\{0,1, \ldots, n\}: \pi_{p q}>\pi_{\bar{p} q} \alpha(k, n)+\pi_{p \bar{q}} \alpha(n, k)\right\}, \\
& h_{q}=\min \left\{k \in\{0,1, \ldots, n\}: \pi_{p q}>\pi_{\bar{p} q} \alpha(n, k)+\pi_{p \bar{q}} \alpha(k, n)\right\} .
\end{aligned}
$$

One can show that $h_{p}\left[h_{q}\right]$ has a simple interpretation. Given that all voters hold $q$ $(p)$ type, it is the number of voters with evidence for $p(q)$ it minimally takes for the decision $p q$ to be the efficient decision. The next theorem characterizes the necessary and sufficient conditions for a quota rule with exception to be the voting rule making informative voting efficient.

Theorem 10 Assume consequentialist preferences. There exists a quota rule with exception making informative voting efficient if and only if $\pi_{p q}>\pi_{\bar{p} q} \alpha\left(h_{p}, h_{q}\right)+$ $\pi_{p \bar{q}} \alpha\left(h_{q}, h_{p}\right)$. In this case, this rule has uniquely defined thresholds $h_{p}$ and $h_{q}$ and an exception rule satisfying

$$
n_{r}^{\mathbf{v}}<m_{r} \text { for both } r \in\{p, q\} \Longrightarrow f(\mathbf{v}) \in\{\bar{p} q, p \bar{q}\} .
$$

\section{Conclusion}

I consider a model where a group of voters with common interests and private information wants to form collective judgments over two propositions which are mutually interconnected. I study the problem of full efficient information aggregation in a Bayesian voting game setting.

I study different settings. To start with, I assume that the evidence for each issue is distributed independently, which may lead to conflicting information. More precisely, one may obtain type $\bar{p} \bar{q}$ while this is not a possible state. In the case of multiple interconnected issues, it is sensible that conflicting information occurs, especially when voters collect information from different resources or form opinions based on independently collected evidence. This leaves room for flexibility in extending the notion of informative strategy. I consider several natural definitions and show that, in almost all cases, there is no voting rule making informative voting efficient. I also consider a setting where instead signals for each proposition that constitute types are distributed jointly, and, information conflicts no longer occur within voters. Under this assumption, there is always a voting rule making informative voting efficient. As the negative 
message mentioned above is led by the impossibility of revealing the inconsistent type, this paper contributes to two other fields: (i) the field of mechanism design that focuses on communication constraints (e.g. Blumrosen and Feldman 2013, Van Zandt 2007), (ii) the field of information aggregation focusing on the consequences of mismatch of type and state spaces (e.g., Schmitz and Tröger 2012, Barelli, Bhattacharya and Siga 2018, Bozbay and Peters 2018). The analysis of the benchmark case is relevant and of particular interest, and I propose abstention as a way to escape from the impossibility. I show that when abstention is a possible strategy, efficient information aggregation can be achieved even when there are information conflicts.

An interesting extension of this paper's analysis might be allowing for pre-voting deliberation together with some degree of diversity in preferences. (See Austen-Smith and Feddersen, 2006, for a model of deliberation in a similar setting.) I consider two issues, which is the simplest case for multi-issue problems. I study exhaustively all possible interconnections between two issues (except bi-implication which is a trivial case). Such agendas are important in practice as examples show. The negative conclusion of Theorem 1 can be extended to an arbitrary number of interconnected issues but extending the complete analysis to many-issue case is not trivial. The number of possible types of interconnections and correspondingly, the number of ways to obtain a group action both grow rapidly with the number of issues, and it is not clear how to obtain general positive results.

\section{A Appendix}

\section{A.1 Properties of a voting rule: an illustration}

As the primary goal of this paper is designing a mechanism, I now introduce some desirable properties of a voting rule to characterize a sub-class of voting rules given in Proposition 1. This section does not aim to provide a throughout axiomatic analysis of all voting rules mentioned in the main text, but to illustrate in the setting of Section 3.4 which desirable properties of voting rules can be attained when issues are interconnected while information is aggregated efficiently. I define anonymity, monotonicity and neutrality as follows:

- Anonymity: For all voting profiles $\left(v_{1}, \ldots, v_{n}\right) \in \mathcal{J}^{n}$ and all permutations $\left(i_{1}, \ldots\right.$, $\left.i_{n}\right)$ of the voters, $f\left(v_{i_{1}}, \ldots, v_{i_{n}}\right)=f\left(v_{1}, \ldots, v_{n}\right)$.

- Monotonicity: For all voting profiles $\mathbf{v}, \mathbf{v}^{\prime} \in \mathcal{J}^{n}$, if for each $r$ in $f(\mathbf{v})$ the voters who accept $r$ in $\mathbf{v}$ also accept $r$ in $\mathbf{v}^{\prime}$, then $f\left(\mathbf{v}^{\prime}\right)=f(\mathbf{v})$. This property implies that increasing the support for the accepted proposition does not reverse the acceptance.

- Neutrality: For all voting profiles $\mathbf{v}, \mathbf{v}^{\prime} \in \mathcal{J}^{n}$ for which there is no permutation $\left(i_{1}, \ldots, i_{n}\right)$ of the voters with $\left(v_{i_{1}}, \ldots, v_{i_{n}}\right)=\left(v_{1}^{\prime}, \ldots, v_{n}^{\prime}\right)$, if for every voter $i$ the vote $v_{i}$ contains $p$ if and only if the vote $v_{i}^{\prime}$ contains $q$, then $f(\mathbf{v})$ contains $p$ if and only if $f\left(\mathbf{v}^{\prime}\right)$ contains $q$. Informally, the two issues are treated symmetrically. 
While some of the voting rules described in Proposition 1 are anonymous, monotonic and neutral, others fail to satisfy any of these properties. Once I impose these axioms, I restrict the large class of rules to those satisfying the following four conditions as I state in the next proposition. For each $\mathbf{v} \in \mathcal{J}^{n}$,

$$
\begin{aligned}
& f(\mathbf{v})=p q \Longleftrightarrow n_{p}^{\mathbf{v}}=n_{q}^{\mathbf{v}}=n \\
& f(\mathbf{v})=p \bar{q} \text { if } n_{p}^{\mathbf{v}}>n_{q}^{\mathbf{v}} \\
& f(\mathbf{v})=\bar{p} q \text { if } n_{p}^{\mathbf{v}}<n_{q}^{\mathbf{v}} \\
& f(\mathbf{v}) \in\{p \bar{q}, \bar{p} q\} \text { if } n_{p}^{\mathbf{v}}=n_{q}^{\mathbf{v}}<n .
\end{aligned}
$$

Proposition 4 Assume consequentialist preferences and that informative voting is efficient with some voting rule. A voting rule $f: \mathcal{J}^{n} \rightarrow \mathcal{J}$ is anonymous, monotonic, neutral and makes informative voting efficient if and only if it belongs to the class of rules defined by (17)-(20).

Proof. Consider a voting rule $f: \mathcal{J}^{n} \rightarrow \mathcal{J}$. Let $n_{r}^{\mathbf{v}}$ denote the number of occurrences of $r \in\{p, q\}$ in a voting profile $\mathbf{v}$.

A. First, let $f$ be defined by (17)-(20). Clearly, $f$ is anonymous. It follows from Proposition 1 that informative voting is efficient with $f$ since for all $\mathbf{v} \in \mathcal{J}^{n}, f(\mathbf{v})=p q$ if and only if $n_{p}^{\mathbf{v}}=n_{q}^{\mathbf{v}}=n$; so, if and only if $\mathbf{v}=(p q, \ldots, p q)$. To show monotonicity of $f$, take two voting profiles $\mathbf{v}, \mathbf{v}^{\prime} \in \mathcal{J}^{n}$ such that for all $r \in f(\mathbf{v})$, the voters who vote for $r$ in $\mathbf{v}$ also vote for $r$ in $\mathbf{v}^{\prime}$.

Case 1: $f(\mathbf{v})=p q$. Then $\mathbf{v}=(p q, \ldots, p q)$. By definition, $\mathbf{v}^{\prime}=\mathbf{v}$ and $f\left(\mathbf{v}^{\prime}\right)=p q$.

Case 2: $f(\mathbf{v})=p \bar{q}$. The definition of $f$ implies either $n_{p}^{\mathbf{v}}>n_{q}^{\mathbf{v}}$ or $n_{p}^{\mathbf{v}}=n_{q}^{\mathbf{v}}<n$; and the definition of $\mathbf{v}^{\prime}$ implies $n_{p}^{\mathbf{v}^{\prime}} \geq n_{p}^{\mathbf{v}}$ and $n_{q}^{\mathbf{v}^{\prime}} \leq n_{q}^{\mathbf{v}}$. Suppose $n_{p}^{\mathbf{v}}>n_{q}^{\mathbf{v}}$. Then, $n_{p}^{\mathbf{v}^{\prime}}>n_{q}^{\mathbf{v}^{\prime}}$ and $f\left(\mathbf{v}^{\prime}\right)=p \bar{q}$. Next, suppose $n_{p}^{\mathbf{v}}=n_{q}^{\mathbf{v}}<n$. If $\mathbf{v}^{\prime} \neq \mathbf{v}$, one has $n_{p}^{\mathbf{v}^{\prime}}>n_{p}^{\mathbf{v}}$ or $n_{q}^{\mathbf{v}^{\prime}}<n_{q}^{\mathbf{v}}$ which means $n_{p}^{\mathbf{v}^{\prime}}>n_{q}^{\mathbf{v}^{\prime}}$ and $f\left(\mathbf{v}^{\prime}\right)=p \bar{q}$.

Case 3: $f(\mathbf{v})=\bar{p} q$. One can show that $f\left(\mathbf{v}^{\prime}\right)=\bar{p} q$ analogously to Case 2.

It remains to show neutrality of $f$. Take two voting profiles $\mathbf{v}, \mathbf{v}^{\prime} \in \mathcal{J}^{n}$ such that $\mathbf{v}_{r}=\mathbf{v}^{\prime}{ }_{r^{\prime}}$ for every distinct $r, r^{\prime} \in\{p, q\}$ and there is no permutation of voters $\left(i_{1}, \ldots, i_{n}\right)$ with $\left(v_{i_{1}}, \ldots, v_{i_{n}}\right)=\left(v_{1}^{\prime}, \ldots, v_{n}^{\prime}\right)$. I have to show that $\left(^{*}\right) f$ accepts $r$ in $\mathbf{v}$ if and only if $f$ accepts $r^{\prime}$ in $\mathbf{v}^{\prime}$. I distinguish 3 cases:

Case 1: $f(\mathbf{v})=p q$. It is clear that $\mathbf{v}^{\prime}=\mathbf{v}$, and $f\left(\mathbf{v}^{\prime}\right)=p q$.

Case 2: $f(\mathbf{v})=p \bar{q}$. By definition of $f$, either $n_{p}^{\mathbf{v}}>n_{q}^{\mathbf{v}}$ or $n_{p}^{\mathbf{v}}=n_{q}^{\mathbf{v}}<n$. One can see that the latter is not possible since then one could find a permutation of voters $\left(i_{1}, \ldots, i_{n}\right)$ with $\left(v_{i_{1}}, \ldots, v_{i_{n}}\right)=\left(v_{1}^{\prime}, \ldots, v_{n}^{\prime}\right)$. Suppose $n_{p}^{\mathbf{v}}>n_{q}^{\mathbf{v}}$. By definition of $\mathbf{v}^{\prime}$, whenever $p(q)$ is accepted in $\mathbf{v}, q(p)$ is accepted in $\mathbf{v}^{\prime}$. This means $n_{p}^{\mathbf{v}^{\prime}}<n_{q}^{\mathbf{v}^{\prime}}$ and $f\left(\mathbf{v}^{\prime}\right)=\bar{p} q$. So, $f$ accepts $p$ in $\mathbf{v}$ and $q$ in $\mathbf{v}^{\prime}$, and it accepts $\bar{q}$ in $\mathbf{v}$ and $\bar{p}$ in $\mathbf{v}^{\prime}$. Hence, $(*)$ holds. 
Case 3: $f(\mathbf{v})=\bar{p} q$. One can show that $f\left(\mathbf{v}^{\prime}\right)=\bar{p} q$ analogously to Case 2 .

B. Conversely, let $f$ be anonymous, monotonic and neutral, and make informative voting efficient. I have to show that $(*) f$ is defined by (17)-(20). By Proposition 1 and informative voting being efficient, $f(\mathbf{v})=p q$ if and only if $\mathbf{v}=(p q, \ldots, p q)$, equivalently $n_{p}^{\mathbf{v}}=n_{q}^{\mathbf{v}}=n$. Now, take a voting profile $\mathbf{v} \in \mathcal{J}^{n} \backslash\{(p q, \ldots, p q)\}$.

Case 1: $n_{p}^{\mathbf{v}}>n_{q}^{\mathbf{v}}$. Suppose for a contradiction, $f(\mathbf{v})=\bar{p} q$. Let $\mathbf{v}^{\prime}$ be a voting profile with $n_{p}^{\mathbf{v}^{\prime}}=n_{q}^{\mathbf{v}}$ and $n_{q}^{\mathbf{v}^{\prime}}=n_{p}^{\mathbf{v}}$. I start by proving the following claim.

Claim: For each combination of $k, l \in\{0, \ldots, n\}$, there is only one voting profile $\mathbf{v} \in \mathcal{J}^{n}$ with $n_{p}^{\mathbf{v}}=k$ and $n_{p}^{\mathbf{v}}=l$ up to the permutations of votes.

The claim follows from the fact that all votes containing $\bar{p}$ are $\bar{p} q$, and similarly, all votes containing $\bar{q}$ are $p \bar{q}$. Hence, subtracting number of $p(q)$ occurrences in a profile from $n$ gives the exact number of $\bar{p} q(p \bar{q})$ votes. Then, there is only one voting profile with $n_{p}^{\mathbf{v}}$ times $q$ and $n_{q}^{\mathbf{v}}$ times $p$ up to permutations of votes. Hence, by neutrality and anonymity, $f\left(\mathbf{v}^{\prime}\right)=p \bar{q}$. However, by monotonicity of $f, f\left(\mathbf{v}^{\prime}\right)=\bar{p} q$ since $n_{p}^{\mathbf{v}^{\prime}} \leq n_{p}^{\mathbf{v}}$ and $n_{q}^{\mathbf{v}^{\prime}} \geq n_{q}^{\mathbf{v}}$, a contradiction. Then, $f(\mathbf{v})=p \bar{q}$.

Case 2: $n_{p}^{\mathbf{v}}<n_{q}^{\mathbf{v}}$. One can show that $f(\mathbf{v})=\bar{p} q$ analogously to Case 1 .

Case 3: $n_{p}^{\mathbf{v}}=n_{q}^{\mathbf{v}}<n$. By Proposition 1 and informative voting being efficient, $f(\mathbf{v}) \in\{p \bar{q}, \bar{p} q\}$.

So, $(*)$ is true.

Among the efficient aggregation possibilities, anonymity, monotonicity and neutrality can be attained. Note that the property of independence, which imposes that the decision on each proposition $r \in\{p, q\}$ only depends on the votes on $r$, is not satisfied by any rule defined in Proposition 4. This rules out quota rules which are very common in the literature and which, given the optimal acceptance thresholds, almost always lead to efficient information aggregation when issues are independent or when there is a single issue. Quota rules are monotonic, anonymous and independent, but not necessarily neutral. Whenever the acceptance thresholds for propositions are equal, they turn out to be neutral.

\section{A.2 Proofs}

I introduce some preliminary derivations before I prove the main results. For the case where types for each proposition are distributed independently (as in Sections 3 and 4), the probability of the three states in $\mathcal{J}$ conditional on the full information $\mathbf{t} \in \mathcal{J}^{n}$ is given as follows, where $k$ is the number of types that contain $p$ in $\mathbf{t}$ and $l$ is the number of types that contain $q$ in $\mathbf{t}$. 


$$
\begin{aligned}
& \operatorname{Pr}(p q \mid \mathbf{t})=\frac{\pi_{p q} a_{p}^{k}\left(1-a_{p}\right)^{n-k} a_{q}^{l}\left(1-a_{q}\right)^{n-l}}{\operatorname{Pr}(\mathbf{t})} \\
& \operatorname{Pr}(p \bar{q} \mid \mathbf{t})=\frac{\pi_{p \bar{q}} a_{p}^{k}\left(1-a_{p}\right)^{n-k}\left(1-a_{\bar{q}}\right)^{l} a_{\bar{q}}^{n-l}}{\operatorname{Pr}(\mathbf{t})} \\
& \operatorname{Pr}(\bar{p} q \mid \mathbf{t})=\frac{\pi_{\bar{p} q}\left(1-a_{\bar{p}}\right)^{k} a_{\bar{p}}^{n-k} a_{q}^{l}\left(1-a_{q}\right)^{n-l}}{\operatorname{Pr}(\mathbf{t})} .
\end{aligned}
$$

The following derivations are for the setting in Section 5, where types for every proposition are distributed jointly. Let $n_{s}^{\mathrm{t}}$ denote the number of occurrences of $s \in \mathcal{J}$ in a type profile $\mathbf{t}$. The notation $n_{s}^{\mathbf{v}}$ is defined similarly for each $s \in \mathcal{J}$ and a voting profile $\mathbf{v}$. Accordingly, the probability of the three states in $\mathcal{J}$ conditional on the full information $\mathbf{t} \in \mathcal{J}^{n}$ is given as follows, where $k=n_{p q}^{\mathbf{t}}$ and $l=n_{\bar{p} q}^{\mathbf{t}}$ (and consequently, $\left.n_{p \bar{q}}^{\mathrm{t}}=n-k-l\right)$.

$$
\begin{aligned}
& \operatorname{Pr}(p q \mid \mathbf{t})=\frac{\pi_{p q} a^{k}(1-a)^{n-k}}{\operatorname{Pr}(\mathbf{t})} \\
& \operatorname{Pr}(\bar{p} q \mid \mathbf{t})=\frac{\pi_{\bar{p} q} a^{l}(1-a)^{n-l}}{\operatorname{Pr}(\mathbf{t})} \\
& \operatorname{Pr}(p \bar{q} \mid \mathbf{t})=\frac{\pi_{p \bar{q}} a^{n-k-l}(1-a)^{k+l}}{\operatorname{Pr}(\mathbf{t})} .
\end{aligned}
$$

Proof of Theorem 1. To start with, I introduce some notation. Given a voting profile $\mathbf{v}$, let $\Theta(\mathbf{v})$ denote the set of all type profiles which possibly lead to $\mathbf{v}$ under informative voting. Let $\sigma$ be an informative strategy for each voter. For informative voting to be efficient, for each $\mathbf{v}$ there must be a common efficient decision $d^{*}$ for all $\mathbf{t} \in \Theta(\mathbf{v})$. This is a very strong requirement since any state in a voting profile $\mathbf{v}$ might come from an equivalent type or $\bar{p} \bar{q}$. Specifically, consider the type profile $\mathbf{t}^{0}=(\bar{p} \bar{q}, \ldots, \bar{p} \bar{q})$. When voters vote informatively, this type profile might lead to any $\mathbf{v} \in \mathcal{J}^{n}$. So, one could only reach efficient outcomes if the same decision(s) is efficient for all type profiles, which is a degenerate case that is ruled out.

Proof of Theorem 2. To start with, I introduce some notation. Given a voting profile $\mathbf{v}$, let $\Theta(\mathbf{v})$ denote the set of all type profiles which possibly lead to $\mathbf{v}$ under informative voting of a given class. Given a type profile $\mathbf{t}$, let $\Omega(\mathbf{t})$ denote the set of all voting profiles which possibly result from $\mathbf{t}$ under informative voting of a given class. Hence we obtain $\Theta(\mathbf{v})(\Omega(\mathbf{t}))$ from $\mathbf{v}(\mathbf{t})$ for a given class of informative voting. Consider a voting rule $f: \mathcal{J}^{n} \rightarrow \mathcal{J}$.

(i) For this part of the proof, assume informative voting of class 1. Whenever I refer to informative voting, this is the class I am referring to. First, let joint efficiency 1 
hold. Suppose there is an exogenously given ordering of judgment sets, and let $f$ be the following voting rule: for all $\mathbf{v} \in \mathcal{J}^{n}, f(\mathbf{v})=d \Leftrightarrow d$ is the highest ordered decision among all decisions which are efficient for some $\mathbf{t} \in \Theta(\mathbf{v})$. Consider any type profile $\hat{\mathbf{t}} \in \mathcal{T}^{n}$ and suppose informative voting. I want to show that $(*)$ for each $\mathbf{v} \in \Omega(\hat{\mathbf{t}})$, $f(\mathbf{v})$ is efficient for $\hat{\mathbf{t}}$. Let $\mathbf{v} \in \Omega(\hat{\mathbf{t}})$. One can show that all type profiles in $\Theta(\mathbf{v})$ share the same subvector restricted to $p q$. (This is because under informative voting all voters with type $p q$ - and only voters with type $p q$ - vote for $p q$, hence all type profiles possibly leading to $\mathbf{v}$ must have the same set of voters with type $p q$.) Since joint efficiency 1 holds, there is some decision $d$ which is efficient for all $\mathbf{t} \in \Theta(\mathbf{v})$, including $\hat{\mathbf{t}}$. By No efficiency ties, if any other decision $d^{\prime} \neq d$ is efficient for some $\mathbf{t} \in \Theta(\mathbf{v})$, it must have the same utility as $d$, which implies that it is efficient for all $\mathbf{t} \in \Theta(\mathbf{v})$. Then, $(*)$ holds.

Conversely, let $f$ make informative voting efficient. Let $\mathbf{t}, \mathbf{t}^{\prime}$ be two type profiles in $\mathcal{T}^{n}$ with $\left\{i: t_{i}=p q\right\}=\left\{i: t_{i}^{\prime}=p q\right\}$. One has to show that $(* *)$ there is $d \in \mathcal{J}$ which is efficient for both $\mathbf{t}, \mathbf{t}^{\prime}$. By construction, for each $\mathbf{v} \in \Omega(\mathbf{t}), \mathbf{t}^{\prime} \in \Theta(\mathbf{v})$; and similarly, for each $\mathbf{v}^{\prime} \in \Omega\left(\mathbf{t}^{\prime}\right), \mathbf{t} \in \Theta\left(\mathbf{v}^{\prime}\right)$. Then, $f(\mathbf{v})$ must be efficient for $\mathbf{t}^{\prime}$ (as well as $\mathbf{t}$ ) and $f\left(\mathbf{v}^{\prime}\right)$ must be efficient for $\mathbf{t}$ (as well as $\mathbf{t}^{\prime}$ ) since informative voting is efficient. So, $(* *)$ holds.

(ii) Assume informative voting of class 2 (3). Let joint efficiency 2 hold. Let $D_{\mathbf{t}} \subseteq \mathcal{J}$ denote the set of efficient decisions given a type profile $\mathbf{t}$. Consider any type profile $\mathbf{t} \in \mathcal{T}^{n}$ and let $\mathbf{v}=\boldsymbol{\sigma}^{\text {inf }}(\mathbf{t})$. Take the type profile $\mathbf{t}^{\prime}$ with $\mathbf{t}^{\prime}=\mathbf{v}$. This implies $\mathbf{t}^{\prime}$ does not have any type $\bar{p} \bar{q}$ in it as a voting profile is free from conflicting types. By construction, we have $\boldsymbol{\sigma}^{i n f}(\mathbf{t})=\boldsymbol{\sigma}^{i n f}\left(\mathbf{t}^{\prime}\right)$. As joint efficiency 2 holds, there must be a decision $d$ that is efficient for both $\mathbf{t}, \mathbf{t}^{\prime}$. (If $\mathbf{t}=\mathbf{t}^{\prime}$, this is straightforward.) By No efficiency ties, if any other decision $d^{\prime} \neq d$ is efficient for (without loss of generality) $\mathbf{t}^{\prime}$, it must necessarily have the same utility as $d$ and be efficient for $\mathbf{t}$ as well. This implies $D_{\mathbf{t}}=D_{\mathbf{t}^{\prime}}$ for all $\mathbf{t}, \mathbf{t}^{\prime} \in \Theta(\mathbf{v})$. Suppose there is an exogenously given ordering of judgment sets, and let $f$ be the following voting rule: for all $\mathbf{v} \in \mathcal{J}^{n}$, $f(\mathbf{v})=d \Longleftrightarrow d$ is the highest ordered decision among all decisions in $D_{\mathbf{t}} \subseteq \mathcal{J}$ for $\mathbf{t}=\mathbf{v}$. This rule clearly renders informative voting of class $2(3)$ efficient.

It is straightforward to show the converse implication. Let $f$ make informative voting of class 2 (3) efficient. Let $\mathbf{t}, \mathbf{t}^{\prime} \in \mathcal{T}^{n}$ be two type profiles with $\boldsymbol{\sigma}^{\text {inf }}(\mathbf{t})=\boldsymbol{\sigma}^{\text {inf }}\left(\mathbf{t}^{\prime}\right)=\mathbf{v}$. Since $f$ makes informative voting efficient, $d=f(\mathbf{v})$ must be efficient for both $\mathbf{t}, \mathbf{t}^{\prime}$.

Proof of Theorem 3. Assume simple preferences. By Theorem 2, it is sufficient to show that $(*)$ joint efficiency 1 never holds.

Suppose for a contradiction, joint efficiency 1 holds. Consider the two type profiles $\mathbf{t}=(p \bar{q}, \ldots, p \bar{q})$ and $\mathbf{t}^{\prime}=(\bar{p} q, \ldots, \bar{p} q)$. Since $\left\{i: t_{i}=p q\right\}=\left\{i: t_{i}^{\prime}=p q\right\}=\emptyset$ and joint efficiency 1 holds, there is a decision which is efficient for both profiles. The judgment set $p \bar{q}$ must be efficient for $\mathbf{t}$ since $p \bar{q}$ must be efficient for some type profile by Nondegeneracy assumption and $\mathbf{t}$ is the type profile that provides the strongest evidence possible in favor of $p \bar{q}$. So, if $p \bar{q}$ was not efficient for $\mathbf{t}$, it would not be efficient for any 
type profile which contradicts to Non-degeneracy assumption. Similarly, $\bar{p} q$ must be efficient for $\mathbf{t}^{\prime}$. Hence, $p \bar{q}$ and $\bar{p} q$ are both efficient given $\mathbf{t}$ or $\mathbf{t}^{\prime}$, which contradicts to No efficiency ties assumption under simple preferences. Hence, we have $\left({ }^{*}\right)$.

Proof of TheOrem 4. Let the condition that $p q$ is efficient only for the unanimous type profile $\mathbf{t}=(p q, \ldots, p q)$ be denoted by $\left(^{*}\right)$. I generally write informative voting for informative voting of class 1 throughout the proof.

A. I first prove that $\left(^{*}\right)$ implies that there is a voting rule for which informative voting is efficient and $A, B>\pi_{p q}$. Assume $\left(^{*}\right)$ holds. This means for every type profile different than the unanimous type profile $(p q, \ldots, p q)$, both $p \bar{q}$ and $\bar{p} q$ are efficient. (Note that under consequentialist preferences, a voter is indifferent between $p \bar{q}$ and $\bar{p} q$.) Then, joint efficiency 1 holds because for every pair of type profiles which share the same set of voters with type $p q$, the requirement of joint efficiency 1 that there is a common efficient decision is satisfied. By Theorem 2, there is a voting rule which makes informative voting efficient. Let $\mathbf{t}, \mathbf{t}^{\prime}$ be type profiles with one $p \bar{q}$ and one $\bar{p} q$ respectively while each of the rest of the types is $p q$. Without loss of generality, let $\mathbf{t}=(p q, \ldots, \bar{p} q)$ and $\mathbf{t}^{\prime}=(p q, \ldots, p \bar{q})$. Using $\left.(21), 22\right)$ and (23) and that $p \bar{q}$ is efficient, I can write the following:

$$
\begin{aligned}
& E(u(p \bar{q}, S) \mid \mathbf{t})>E(u(p q, S) \mid \mathbf{t}) \\
\Leftrightarrow & \pi_{p \bar{q}} a_{p}^{n-1}\left(1-a_{p}\right)\left(1-a_{\bar{q}}\right)^{n}+\pi_{\bar{p} q}\left(1-a_{\bar{p}}\right)^{n-1} a_{\bar{p}} a_{q}^{n}>\pi_{p q} a_{p}^{n-1}\left(1-a_{p}\right) a_{q}^{n} \\
\Leftrightarrow & \pi_{p \bar{q}}\left(\frac{1-a_{\bar{q}}}{a_{q}}\right)^{n}+\pi_{\bar{p} q}\left(\frac{1-a_{\bar{p}}}{a_{p}}\right)^{n-1}\left(\frac{a_{\bar{p}}}{1-a_{p}}\right)>\pi_{p q} .
\end{aligned}
$$

Similarly,

$$
\begin{aligned}
& E\left(u(p \bar{q}, S) \mid \mathbf{t}^{\prime}\right)>E\left(u(p q, S) \mid \mathbf{t}^{\prime}\right) \\
\Leftrightarrow & \pi_{p \bar{q}} a_{p}^{n}\left(1-a_{\bar{q}}\right)^{n-1} a_{\bar{q}}+\pi_{\bar{p} q}\left(1-a_{\bar{p}}\right)^{n} a_{q}^{n-1}\left(1-a_{q}\right)>\pi_{p q} a_{p}^{n} a_{q}^{n-1}\left(1-a_{q}\right) \\
\Leftrightarrow & \pi_{p \bar{q}}\left(\frac{1-a_{\bar{q}}}{a_{q}}\right)^{n-1}\left(\frac{a_{\bar{q}}}{1-a_{q}}\right)+\pi_{\bar{p} q}\left(\frac{1-a_{\bar{p}}}{a_{p}}\right)^{n}>\pi_{p q} .
\end{aligned}
$$

So, $A, B>\pi_{p q}$.

B. I now prove that if there is a voting rule for which informative voting is efficient, $\left.{ }^{*}\right)$ holds. Consider a voting rule $f: \mathcal{J}^{n} \rightarrow \mathcal{J}$ and suppose $f$ makes informative voting efficient. By Theorem 2, joint efficiency 1 holds. Given a type profile $\mathbf{t} \in \mathcal{T}^{n}$, let $\Gamma(\mathbf{t})$ denote the set of type profiles which have the same subvector on $p q$ as in $\mathbf{t}$. Now, take a type profile $\hat{\mathbf{t}} \in \mathcal{T}^{n}$ with $k$ times $p q$ where $1 \leq k<n$. The proof proceeds in several steps.

Claim 1: There is a type profile $\mathbf{t} \in \Gamma(\hat{\mathbf{t}})$ with $n_{p}^{\mathbf{t}}=k$ and $n_{q}^{\mathbf{t}}=k$.

Any type profile with $k$ times $p q$ and $n-k$ times $\bar{p} \bar{q}$ satisfies this condition and one of these type profiles is obviously in $\Gamma(\hat{\mathbf{t}})$. Now, take $\tilde{\mathbf{t}} \in \mathcal{T}^{n}$ with $k-1$ times $p q$. 
Claim 2: There is a type profile $\mathbf{t} \in \Gamma(\tilde{\mathbf{t}})$ with $n_{p}^{\mathbf{t}}=k$ and $n_{q}^{\mathbf{t}}=k$.

One can easily see there is always a type profile with the exact same $p q$ structure as $\tilde{\mathbf{t}}$ and with only one occurrence of $p \bar{q}$ and only one occurrence of $\bar{p} q$.

Claim 3: Under consequentialist preferences, for all $\mathbf{t}, \mathbf{t}^{\prime} \in \mathcal{T}^{n}$ with $n_{p}^{\mathbf{t}}=n_{p}^{\mathbf{t}^{\prime}}$ and $n_{q}^{\mathbf{t}}=n_{q}^{\mathbf{t}^{\prime}}, E(u(d, S) \mid \mathbf{t})=E\left(u(d, S) \mid \mathbf{t}^{\prime}\right)$ for each $d \in \mathcal{J}$.

The claim follows from the expressions (21)-(22). By joint efficiency 1, there is a decision $d \in \mathcal{J}$ which is efficient for all $\mathbf{t} \in \Gamma(\hat{\mathbf{t}})$. Similarly, there is a decision $d \in \mathcal{J}$ which is efficient for all $\mathbf{t} \in \Gamma(\tilde{\mathbf{t}})$. Combining Claim 1, 2 and 3 , one obtains that the same decision $d \in \mathcal{J}$ is efficient for all $\mathbf{t} \in \Gamma(\hat{\mathbf{t}})$ and all $\mathbf{t} \in \Gamma(\tilde{\mathbf{t}})$. Since this is true for all $k$ with $1 \leq k<n$, there is a decision $d$ which is efficient for all $\mathbf{t} \in \mathcal{T}^{n} \backslash\{(p q, \ldots, p q)\}$. By Non-degeneracy, $p q$ is efficient for $\mathbf{t}=(p q, \ldots, p q)$. Hence, $d$ must be in $\{p \bar{q}, \bar{p} q\}$ since otherwise $p q$ would be efficient for all type profiles which contradicts Non-degeneracy. Hence, $(*)$ holds.

C. I finally prove that $A, B>\pi_{p q}$ implies (*). Let $A, B>\pi_{p q}$. I first prove the following claim.

Claim 4: The expected utility of $p q$ given a type profile $\mathbf{t}$ is an increasing function of $n_{p}^{\mathrm{t}}$ and $n_{q}^{\mathrm{t}}$.

The claim follows from the definition of the utility function and from $\operatorname{Pr}(S=p q \mid \mathbf{t})$ being an increasing function of $n_{p}^{\mathbf{t}}$ and $n_{q}^{\mathbf{t}}$ in (21). Let $\mathbf{t}, \mathbf{t}^{\prime} \in \mathcal{T}^{n}$ be type profiles with one $p \bar{q}$ and one $\bar{p} q$ respectively while each of the rest of the types is $p q$. Without loss of generality, let $\mathbf{t}=(p q, \ldots, \bar{p} q)$ and $\mathbf{t}^{\prime}=(p q, \ldots, p \bar{q})$.

By (27)-(32), one has $E(u(p \bar{q}, S) \mid \mathbf{t})>E(u(p q, S) \mid \mathbf{t})$ and $E\left(u(p \bar{q}, S) \mid \mathbf{t}^{\prime}\right)>E\left(u(p q, S) \mid \mathbf{t}^{\prime}\right)$. By Claim 4, it follows that $E(u(p \bar{q}, S) \mid \mathbf{t})=E(u(\bar{p} q, S) \mid \mathbf{t})>E(u(p q, S) \mid \mathbf{t})$ for all $\mathbf{t} \in \mathcal{T}^{n} \backslash\{(p q, \ldots, p q)\}$ which means $p \bar{q}, \overline{p q}$ are efficient for each $\mathbf{t} \in \mathcal{T}^{n} \backslash\{(p q, \ldots, p q)\}$. Thus, $(*)$ holds.

Proof of Proposition 1. Consider a voting rule $f: \mathcal{J}^{n} \rightarrow \mathcal{J}$. To show the 'if' part, let the rule $f$ be defined as $f(\mathbf{v})=p q$ if $\mathbf{v}=(p q, \ldots, p q)$ and $f(\mathbf{v}) \in\{p \bar{q}, \bar{p} q\}$ for every other $\mathbf{v} \in \mathcal{J}^{n}$. To prove that informative voting is efficient with $f$, I have to show that $p q$ is efficient only for the type profile $\mathbf{t}$ where every type is $p q$, i.e., for $\mathbf{t}=(p q, \ldots, p q)$, since this is the only type profile which gives $\boldsymbol{\sigma}^{\text {inf }}(\mathbf{t})=(p q, \ldots, p q)$ under informative voting of class 1 . For all other type profiles, $p \bar{q}$ and $\bar{p} q$ must both be efficient which follows from Non-degeneracy and Joint efficiency 1 (and also given that $p \bar{q}$ is efficient for $\mathbf{t} \Longleftrightarrow \bar{p} q$ is efficient for $\mathbf{t}$ by $(3))$.

To show converse, let $f$ make informative voting efficient. Note that Theorem $4 \mathrm{im}-$ plies that decision $p q$ is only efficient for the unanimous type profile $\mathbf{t}=(p q, \ldots, p q)$ under consequentialist preferences. Then, for all voting profiles obtained by informative voting from any $\mathbf{t} \in \mathcal{T}^{n} \backslash\{(p q, \ldots, p q)\}, f(\mathbf{v}) \in\{p \bar{q}, \bar{p} q\}$. By Non-degeneracy, $p q$ is efficient for $\mathbf{t}=(p q, \ldots, p q)$. By $f$ making informative voting efficient, $f(\mathbf{v})=p q$ if $\mathbf{v}=(p q, \ldots, p q)$. 
Proof of Theorem 5. Since by (21)-(23) the conditional distribution of the state given full information $\mathbf{t} \in \mathcal{T}^{n}$ depends on $\mathbf{t}$ only via the numbers $n_{p}^{\mathbf{t}}$ and $n_{q}^{\mathbf{t}}$, so does the conditional expected utility of each decision, and hence, the set of efficient decisions. Recall that, for each $(k, l) \in\{0,1, \ldots, n\}^{2}, F(k, l) \in \mathcal{J}$ is a decision that is efficient for some (hence, every) $\mathbf{t} \in \mathcal{T}^{n}$ for which $n_{p}^{\mathbf{t}}=k$ and $n_{q}^{\mathbf{t}}=l$. Under informative voting with abstention, those who abstain have type $\bar{p} \bar{q}$. This means that for any given type profile $\mathbf{t}, n_{p}^{\mathbf{t}}=n_{p}^{\mathbf{v}}$ and $n_{q}^{\mathbf{t}}=n_{q}^{\mathbf{v}}$ if voters vote according to the informative strategy with abstention. The voting rule $f$ defined by $\mathbf{v} \mapsto f(\mathbf{v})=F\left(n_{p}^{\mathbf{v}}, n_{q}^{\mathbf{v}}\right)$ renders informative voting efficient.

Proof of Theorem 6. Consider a rule $f: \mathcal{J}_{\phi}^{n} \rightarrow \mathcal{J}$.

A. First, assume $f$ is the quota rule with exception with thresholds $k_{p}$ and $k_{q}$ and the exception rule (4). Consider a given type profile $\mathbf{t} \in \mathcal{T}^{n}$. Supposing that voters hold informative strategy with abstention, the resulting voting profile has $n_{r}^{\mathbf{v}}=n_{r}^{\mathbf{t}}$ for each $r \in\{p, q\}$ given that voters who abstain have type $\bar{p} \bar{q}$. I have to show that the decision $d=f(\mathbf{v})$ is efficient for $\mathbf{t}$, i.e., that $\left(^{*}\right) E(u(d, S) \mid \mathbf{t})>E\left(u\left(d^{\prime}, S\right) \mid \mathbf{t}\right)$ for all $d^{\prime} \in \mathcal{J} \backslash\{d\}$. (I use ' $>$ ' rather than ' $\geq$ ' in $\left(^{*}\right)$ because of No efficiency ties assumption.)

Case 1: Let $d=p q$. By definition of $f$, I have $n_{p}^{\mathbf{v}} \geq k_{p}$ and $n_{q}^{\mathbf{v}} \geq k_{q}$. Equivalently, $n_{p}^{\mathbf{t}} \geq k_{p}$ and $n_{q}^{\mathbf{t}} \geq k_{q}$. These imply $\frac{\pi_{p q}}{\pi_{\bar{p} q}}>\left(\frac{1-a_{\bar{p}}}{a_{p}}\right)^{n_{p}^{\mathrm{t}}}\left(\frac{a_{\bar{p}}}{1-a_{p}}\right)^{n-n_{p}^{\mathbf{t}}}$ and $\frac{\pi_{p q}}{\pi_{p \bar{q}}}>\left(\frac{1-a_{\bar{q}}}{a_{q}}\right)^{n_{q}^{\mathrm{t}}}\left(\frac{a_{\bar{q}}}{1-a_{q}}\right)^{n-n_{q}^{\mathrm{t}}}$ by (5) and by (6) respectively and by $\beta_{r}$ being a decreasing function of $n_{r}^{\mathbf{t}}$ for each proposition $r$. By (21)-(22), we have $\operatorname{Pr}(p q \mid \mathbf{t})>\operatorname{Pr}(\bar{p} q \mid \mathbf{t})$ and $\operatorname{Pr}(p q \mid \mathbf{t})>\operatorname{Pr}(p \bar{q} \mid \mathbf{t})$ which imply $\left(^{*}\right)$.

Case 2: Let $d=\bar{p} q$. By definition of $f$, this may mean one of the following:

(i) $n_{p}^{\mathbf{v}}<k_{p}$ and $n_{q}^{\mathbf{v}} \geq k_{q}$, or;

(ii) $n_{p}^{\mathbf{v}}<k_{p}, n_{q}^{\mathbf{v}}<k_{q}$, and $\frac{\pi_{p \bar{q}}}{\pi_{\bar{p} q}}<\frac{\beta_{p}\left(n_{p}^{\mathbf{v}}\right)}{\beta_{q}\left(n_{q}^{\mathbf{v}}\right)}$.

Suppose (i). We must then have $\frac{\pi_{p q}}{\pi_{\bar{p} q}}<\left(\frac{1-a_{\bar{p}}}{a_{p}}\right)^{n_{p}^{\mathrm{t}}}\left(\frac{a_{\bar{p}}}{1-a_{p}}\right)^{n-n_{p}^{\mathrm{t}}}$ and $\frac{\pi_{p q}}{\pi_{p \bar{q}}}>\left(\frac{1-a_{\bar{q}}}{a_{q}}\right)^{n_{q}^{\mathrm{t}}}\left(\frac{a_{\bar{q}}}{1-a_{q}}\right)^{n-n_{q}^{\mathrm{t}}}$ by (5) and by (6) respectively (and by $\beta_{r}\left(n_{r}^{\mathbf{t}}\right)$ being a decreasing function of $n_{r}^{\mathbf{t}}$ for each proposition $r)$. These further imply $\operatorname{Pr}(\bar{p} q \mid \mathbf{t})>\operatorname{Pr}(p q \mid \mathbf{t})>\operatorname{Pr}(p \bar{q} \mid \mathbf{t})$, where the former inequality follows from (21) and (23) and the latter inequality follows from (21) and (22). Hence, we have (*). Suppose (ii). We can rewrite $\frac{\pi_{p \bar{q}}}{\pi_{\bar{p} q}}<\frac{\beta_{p}\left(n_{p}^{\mathbf{v}}\right)}{\beta_{q}\left(n_{q}^{\mathbf{v}}\right)}$ as $\frac{\pi_{p \bar{q}}}{\pi_{\bar{p} q}}<\left(\frac{1-a_{\bar{p}}}{a_{p}}\right)^{n_{p}^{\mathrm{t}}}\left(\frac{a_{\bar{p}}}{1-a_{p}}\right)^{n-n_{p}^{\mathrm{t}}}\left(\frac{a_{q}}{1-a_{\bar{q}}}\right)^{n_{q}^{\mathrm{t}}}\left(\frac{1-a_{q}}{a_{\bar{q}}}\right)^{n-n_{q}^{\mathrm{t}}}$. By (22) and (23), this implies $\operatorname{Pr}(\bar{p} q \mid \mathbf{t})>\operatorname{Pr}(p \bar{q} \mid \mathbf{t})$. Note that as $n_{p}^{\mathrm{t}}<k_{p}, n_{q}^{\mathrm{t}}<k_{q}$, by definition of $k_{p}, k_{q}$, the efficient decision cannot be $p q$ as discussed above. Hence, I have $\left(^{*}\right)$.

The case of $d=p \bar{q}$ is analogous to the case of $d=\bar{p} q$. Hence, I have the desired result $(*)$ for each $d \in \mathcal{J}$. 
B. Conversely, suppose informative voting with abstention is efficient under $f$. I consider any $\mathbf{t} \in \mathcal{T}^{n}$ and the corresponding voting profile $\mathbf{v} \in \mathcal{J}_{\phi}^{n}$ assuming informative voting with abstention. Suppose $f(\mathbf{v})=p q$. By efficiency of $p q$, one must have $E(u(p q, S) \mid \mathbf{t})>E\left(u\left(d^{\prime}, S\right) \mid \mathbf{t}\right)$ for all $d^{\prime} \neq p q$, which is equivalent to $\operatorname{Pr}(p q \mid \mathbf{t})>\operatorname{Pr}\left(d^{\prime} \mid \mathbf{t}\right)$ for all $d^{\prime} \neq p q$ under simple preferences. For $d^{\prime}=\bar{p} q$, I can write $\frac{\pi_{p q}}{\pi_{\bar{p} q}}>\left(\frac{1-a_{\bar{p}}}{a_{p}}\right)^{n_{p}^{\mathrm{t}}}\left(\frac{a_{\bar{p}}}{1-a_{p}}\right)^{n-n_{p}^{\mathrm{t}}}$ by (21) and (23). Similarly, by (21) and (22), $\frac{\pi_{p q}}{\pi_{p \bar{q}}}>\left(\frac{1-a_{\bar{q}}}{a_{q}}\right)^{n_{q}^{\mathbf{t}}}\left(\frac{a_{\bar{q}}}{1-a_{q}}\right)^{n-n_{q}^{\mathbf{t}}}$ for $d^{\prime}=p \bar{q}$. As $n_{r}^{\mathbf{v}}=n_{r}^{\mathbf{t}}$ for each $r \in\{p, q\}$, the former implies $n_{p}^{\mathbf{v}} \geq k_{p}$ and the latter implies $n_{q}^{\mathbf{v}} \geq k_{q}$ by the definitions of $k_{p}, k_{q}$ and $\beta_{p}, \beta_{q}$ being decreasing functions of $n_{p}^{\mathbf{v}}, n_{q}^{\mathbf{v}}$ respectively.

Now, let $f(\mathbf{v})=\bar{p} q$. I have to show that (i) or (ii) holds. From $\operatorname{Pr}(\bar{p} q \mid \mathbf{t})>\operatorname{Pr}(p q \mid \mathbf{t})$, I can write the following using (21) and (23): $\frac{\pi_{p q}}{\pi_{\bar{p} q}}<\left(\frac{1-a_{\bar{p}}}{a_{p}}\right)^{n_{p}^{\mathrm{t}}}\left(\frac{a_{\bar{p}}}{1-a_{p}}\right)^{n-n_{p}^{\mathrm{t}}}$, which implies $n_{p}^{\mathbf{t}}=n_{p}^{\mathbf{v}}<k_{p}$ as $k_{p}$ is the minimum value satisfying the reverse inequality and the RHS is decreasing in $n_{p}^{\mathbf{t}}$. By (22) and (23), $\operatorname{Pr}(\bar{p} q \mid \mathbf{t})>\operatorname{Pr}(p \bar{q} \mid \mathbf{t})$ if and only if $\frac{\pi_{p \bar{q}}}{\pi_{\bar{p} q}}<\left(\frac{1-a_{\bar{p}}}{a_{p}}\right)^{n_{p}^{\mathrm{t}}}\left(\frac{a_{\bar{p}}}{1-a_{p}}\right)^{n-n_{p}^{\mathrm{t}}}\left(\frac{a_{q}}{1-a_{\bar{q}}}\right)^{n_{q}^{\mathrm{t}}}\left(\frac{1-a_{q}}{a_{\bar{q}}}\right)^{n-n_{q}^{\mathrm{t}}}$. Since $n_{r}^{\mathbf{v}}=n_{r}^{\mathbf{t}}$ for each $r \in\{p, q\}$ under informative voting with abstention, I can rewrite this expression for $n_{r}^{\mathbf{v}}$ as $(* *)$ $\frac{\pi_{p \bar{q}}}{\pi_{\bar{p} q}}<\left(\frac{1-a_{\bar{p}}}{a_{p}}\right)^{n_{p}^{\mathbf{v}}}\left(\frac{a_{\bar{p}}}{1-a_{p}}\right)^{n-n_{p}^{\mathbf{v}}}\left(\frac{a_{q}}{1-a_{\bar{q}}}\right)^{n_{q}^{\mathbf{v}}}\left(\frac{1-a_{q}}{a_{\bar{q}}}\right)^{n-n_{q}^{\mathbf{v}}}$. The RHS of $(* *)$ is equivalent to $\frac{\beta_{p}\left(n_{p}^{\mathbf{v}}\right)}{\beta_{q}\left(n_{q}^{v}\right)}$. So, if $f(\mathbf{v})=\bar{p} q$, one always has $(* *) \frac{\pi_{p \bar{q}}}{\pi_{\bar{p} q}}<\frac{\beta_{p}\left(n_{p}^{\mathbf{v}}\right)}{\beta_{q}\left(n_{q}^{\mathbf{v}}\right)}$. I have already shown that $n_{p}^{\mathbf{v}}<k_{p}$. If, moreover, $n_{q}^{\mathbf{v}}<k_{q}$, I have (ii). I will show that $(* *)$ necessarily holds when $n_{p}^{\mathbf{v}}<k_{p}$ and $n_{q}^{\mathbf{v}} \geq k_{q}$. Let $n_{q}^{\mathbf{v}} \geq k_{q}$. Then, $\frac{\pi_{p q}}{\pi_{p \bar{q}}}>\beta_{q}\left(n_{q}^{\mathbf{v}}\right)$ by (6). Rearranging, I have $\frac{\pi_{p \bar{q}}}{\pi_{p q}}<1 / \beta_{q}\left(n_{q}^{\mathbf{v}}\right)$. Multiplying by $\frac{\pi_{p q}}{\pi_{\bar{p} q}}<\beta_{p}\left(n_{p}^{\mathbf{v}}\right)$, one obtains $(* *)$. So, if for a voting profile $\mathbf{v}, n_{p}^{\mathbf{v}}<k_{p}$ and $n_{q}^{\mathbf{v}} \geq k_{q}, f(\mathbf{v})$ must be $\bar{p} q$ as it makes informative voting efficent. One can easily check that there is no case other than (i) and (ii) where $\bar{p} q$ is the efficient decision for $\mathbf{t}=\mathbf{v}$. So, if $f(\mathbf{v})=\bar{p} q$, one must have (i) or (ii).

The case of $f(\mathbf{v})=p \bar{q}$ is analogous.

Proof of Theorem 7. A. First, suppose $f: \mathcal{J}_{\phi}^{n} \rightarrow \mathcal{J}$ is a quota rule with exception with thresholds $m_{p}$ and $m_{q}$ and some exception rule, and suppose further that it makes informative voting efficient. I have to show that

(*) $m_{p}=l_{p}$ and $m_{q}=l_{q}$,

(**) $n_{r}^{\mathbf{v}}<l_{r}$ for each $r \in\{p, q\} \Longrightarrow f(\mathbf{v}) \in\{p \bar{q}, \bar{p} q\}$, and

$(* * *) \gamma\left(l_{p}, l_{q}\right)>1 / 2$.

Consider a type profile $\mathbf{t} \in \mathcal{T}^{n}$. Assuming informative voting with abstention, the resulting voting profile $\mathbf{v}$ has the same number of $p$ and $q$ occurrences as $\mathbf{t}$, thus, $n_{p}^{\mathbf{v}}=n_{p}^{\mathbf{t}}$ and $n_{q}^{\mathbf{v}}=n_{q}^{\mathbf{t}}$ (as those who abstain have type $\bar{p} \bar{q}$ ).

To show $\left({ }^{*}\right)$, suppose $n_{p}^{\mathbf{t}}=n$ and $n_{q}^{\mathbf{t}}=l_{q}$. By $(9), \gamma\left(n, l_{q}\right)>1 / 2$. So $f(\mathbf{v})=p q$ by Proposition 2. Thus, $l_{q} \geq m_{q}$ as $f$ is a quota rule with exception. Similarly, it can be shown that $l_{p} \geq m_{p}$. I now have to show the converse inequalities. Consider a voting 
profile $\mathbf{v} \in \mathcal{J}_{\phi}^{n}$ for which $n_{p}^{\mathbf{v}}=m_{p}$ and $n_{q}^{\mathbf{v}}=n$ which is greater than or equal to $m_{q}$. The resulting decision is $f(\mathbf{v})=p q$ by definition of $f$. Since $f$ makes informative voting with abstention efficient, $\gamma\left(n_{p}^{\mathbf{v}}, n_{q}^{\mathbf{v}}\right)=\gamma\left(m_{p}, n\right)>1 / 2$ by Proposition 2. Hence, $m_{p} \geq l_{p}$ by definition of $l_{p}$. Analogously, one shows that $m_{q} \geq l_{q}$. So, I have the desired result $(*)$.

To show (**), suppose $n_{r}^{\mathbf{v}}<l_{r}$ for each $r \in\{p, q\}$ (note that if $l_{r}=0$ for any $r$, we can omit this stage as the premise of $\left({ }^{* *}\right)$ is never satisfied.) Recall that $n_{r}^{\mathbf{v}}=n_{r}^{\mathbf{t}}$ for each $r \in\{p, q\}$ under informative voting with abstention, By definition of $l_{p}$ and $l_{q}$, I must have $\gamma\left(n_{p}^{\mathbf{t}}, n_{q}^{\mathbf{t}}\right)<1 / 2$ since $\gamma$ is increasing in both arguments and $n_{r}^{\mathbf{t}}<l_{r}$ for each $r$. So, $p \bar{q}$ and $\bar{p} q$ are efficient, and as $f$ makes informative voting efficient, $f(\mathbf{v}) \in\{p \bar{q}, \bar{p} q\}$.

Finally, I need to show (***). For any voting profile $\mathbf{v} \in \mathcal{J}_{\phi}^{n}$ for which $n_{p}^{\mathbf{v}}=l_{p}\left(=m_{p}\right)$ and $n_{q}^{\mathbf{v}}=l_{q}\left(=m_{q}\right)$, I have $f(\mathbf{v})=p q$ by definition of $f$, so that by Proposition 2, $\gamma\left(n_{p}^{\mathbf{v}}, n_{q}^{\mathbf{v}}\right)>1 / 2$, i.e., $\gamma\left(l_{p}, l_{q}\right)>1 / 2$.

B. Conversely, let $\gamma\left(l_{p}, l_{q}\right)>1 / 2$. I now show that the quota rule with exception $f$ with thresholds $l_{p}$ and $l_{q}$ and the exception rule (10) makes informative voting efficient. I start by proving that for all $k, l \in\{0, \ldots, n\}$,

$$
\gamma(k, l)>1 / 2 \Leftrightarrow\left[k \geq l_{p} \text { and } l \geq l_{q}\right]
$$

If $k \geq l_{p}$ and $l \geq l_{q}$, then $\gamma(k, l) \geq \gamma\left(l_{p}, l_{q}\right)>1 / 2$, where the first inequality holds because $\gamma$ is increasing in each argument. If $k<l_{p}$, then $\gamma(k, l) \leq \gamma(k, n) \leq 1 / 2$, where the last inequality holds by definition of $l_{p}(>k)$. Analogously, if $l \leq l_{q}$, then $\gamma(k, l) \leq 1 / 2$.

Now consider any type profile $\mathbf{t} \in \mathcal{T}^{n}$. Assuming informative voting with abstention, the resulting voting profile $\mathbf{v}$ has the same number of $p$ and $q$ occurrences as $\mathbf{t}$, thus, $n_{p}^{\mathbf{v}}=n_{p}^{\mathbf{t}}$ and $n_{q}^{\mathbf{v}}=n_{q}^{\mathbf{t}}$. I must show that $f(\mathbf{v})$ is efficient for $\mathbf{t}$. First, if $n_{p}^{\mathbf{t}} \geq l_{p}$ and $n_{q}^{\mathbf{t}} \geq l_{q}$, the decision is $f(\mathbf{v})=p q$, which is efficient by Proposition 2 since $\gamma\left(n_{p}^{\mathbf{t}}, n_{q}^{\mathbf{t}}\right)>1 / 2$ by (33). Second, if $n_{p}^{\mathbf{t}}<l_{p}$ or $n_{q}^{\mathbf{t}}<l_{q}$, the resulting decision $f(\mathbf{v})$ is in $\{\bar{p} q, p \bar{q}\}$, which is efficient by Proposition 2 since $\gamma\left(n_{p}^{\mathbf{t}}, n_{q}^{\mathbf{t}}\right) \leq 1 / 2$ by (33).

Proof of Theorem 8. By (24)-(26) the conditional distribution of the state given full information $\mathbf{t} \in \mathcal{J}^{n}$ depends on $\mathbf{t}$ only via the numbers $n_{p q}^{\mathbf{t}}$ and $n_{\bar{p} q}^{\mathbf{t}}$, so does the conditional expected utility of each decision, and hence, the set of efficient decisions. Take any type profile $\mathbf{t} \in \mathcal{J}^{n}$. Assume informative voting, so, the resulting voting profile is $\mathbf{v}=\mathbf{t}$. Suppose one can only observe $n_{p}^{\mathbf{v}}$ and $n_{q}^{\mathbf{v}}$. As only those voting for $\bar{p} q$ vote $\bar{p}$, we must have $n_{\bar{p} q}^{\mathbf{v}}=n-n_{p}^{\mathbf{v}}$. Similarly, $n_{p \bar{q}}^{\mathbf{v}}=n-n_{q}^{\mathbf{v}}$ which gives us $n_{p q}^{\mathbf{v}}=$ $n_{p}^{\mathbf{v}}+n_{q}^{\mathbf{v}}-n$. For each $(k, l) \in\{0,1, \ldots, n\}^{2}$, let $\tilde{F}(k, l) \in \mathcal{J}$ be a decision that is efficient for some (hence, every) $\mathbf{t} \in \mathcal{J}^{n}$ for which $n_{p q}^{\mathrm{t}}=k$ and $n_{\bar{p} q}^{\mathrm{t}}=l$ in the setting of jointly distributed types. The voting rule $f$ defined by $\mathbf{v} \mapsto f(\mathbf{v})=F\left(n_{p}^{\mathbf{v}}+n_{q}^{\mathbf{v}}-n, n-n_{p}^{\mathbf{v}}\right)$ renders informative voting efficient.

Proof of Theorem 9. Consider a voting rule $f: \mathcal{J}^{n} \rightarrow \mathcal{J}$. 
A. First, assume that $f$ is the rule given by (13). Take any type profile $\mathbf{t} \in \mathcal{J}^{n}$ and suppose informative voting. Then, the resulting voting profile is $\mathbf{v}=\mathbf{t}$. I have to show that $d=f(\mathbf{v})$ is efficient for $\mathbf{t}$, hence $E(u(d, S) \mid \mathbf{t})>E\left(u\left(d^{\prime}, S\right) \mid \mathbf{t}\right)$ for all $d^{\prime} \in \mathcal{J} \backslash\{d\}$. This is equivalent to showing that $\left(^{*}\right) \operatorname{Pr}(d \mid \mathbf{t})>\operatorname{Pr}\left(d^{\prime} \mid \mathbf{t}\right)$ for all $d^{\prime} \in \mathcal{J} \backslash\{d\}$.

Suppose $d=p q$. By definition of $f$, one must have $\frac{\pi_{p q}}{\pi_{\bar{p} q}}>\alpha\left(n_{p}^{\mathbf{v}}, n_{q}^{\mathbf{v}}\right)$ and $\frac{\pi_{p q}}{\pi_{p \bar{q}}}>$ $\alpha\left(n_{q}^{\mathbf{v}}, n_{q}^{\mathbf{v}}\right)$; or equivalently, $\frac{\pi_{p q}}{\pi_{\bar{p} q}}>\left(\frac{a}{1-a}\right)^{2 n-2 n_{p}^{\mathbf{v}}-n_{q}^{\mathbf{v}}}$ and $\frac{\pi_{p q}}{\pi_{p \bar{q}}}>\left(\frac{a}{1-a}\right)^{2 n-2 n_{q}^{\mathbf{v}}-n_{p}^{\mathbf{v}}}$. Let me focus on the former inequality. As only those voting for $\bar{p} q$ vote $\bar{p}$, we must have $n_{\bar{p} q}^{\mathbf{v}}=n-n_{p}^{\mathbf{v}}$. Similarly, $n_{p \bar{q}}^{\mathbf{v}}=n-n_{q}^{\mathbf{v}}$ which gives us $n_{p q}^{\mathbf{v}}=n_{p}^{\mathbf{v}}+n_{q}^{\mathbf{v}}-n$. Then, I can rewrite $2 n-2 n_{p}^{\mathbf{v}}-n_{q}^{\mathbf{v}}$ as $n-n_{p}^{\mathbf{v}}-\left(n_{p}^{\mathbf{v}}+n_{q}^{\mathbf{v}}-n\right)$, which is equal to $n_{\bar{p} q}^{\mathbf{v}}-n_{p q}^{\mathbf{v}}$. Hence, $\frac{\pi_{p q}}{\pi_{\bar{p} q}}>\left(\frac{a}{1-a}\right)^{n_{\bar{p} q}^{\mathrm{V}}-n_{p q}^{\vee}}$, or equivalently,

$$
\pi_{p q} a^{n_{p q}^{\mathrm{v}}}(1-a)^{-n_{p q}^{\mathrm{v}}}>\pi_{\bar{p} q} a^{n_{\bar{p} q}^{\mathrm{v}}}(1-a)^{-n_{\bar{p} q}^{\mathrm{v}}}
$$

As $\mathbf{v}=\mathbf{t}$, multiplying both sides of $(34)$ by $(1-a)^{n}$, I obtain $\pi_{p q} a^{n_{p q}^{\mathrm{t}}}(1-a)^{n-n_{p q}^{\mathrm{t}}}>$ $\pi_{\bar{p} q} a^{n_{\bar{p} q}^{\mathrm{t}}}(1-a)^{n-n_{\bar{p} q}^{\mathrm{t}}}$, which by (24) and (25), is equivalent to $\left(^{*}\right)$ for $d^{\prime}=\bar{p} q$. The proof for $d^{\prime}=p \bar{q}$ is analogous.

Let $d=\bar{p} q$. By definition of $f$, one must have $\frac{\pi_{p q}}{\pi_{\bar{p} q}}<\alpha\left(n_{p}^{\mathbf{v}}, n_{q}^{\mathbf{v}}\right)$ and $\frac{\pi_{\bar{p} q}}{\pi_{p \bar{q}}}>\beta\left(n_{p}^{\mathbf{v}}, n_{q}^{\mathbf{v}}\right)$; or equivalently, $\frac{\pi_{p q}}{\pi_{\bar{p} q}}<\left(\frac{a}{1-a}\right)^{2 n-2 n_{p}^{\mathrm{v}}-n_{q}^{\mathrm{v}}}$ and $\frac{\pi_{\bar{p} q}}{\pi_{p \bar{q}}}>\left(\frac{a}{1-a}\right)^{n_{p}^{\mathrm{v}}-n_{q}^{\mathrm{v}}}$. I already know that the former can be written as (34) with the opposite sign; so, following the above discussion, $\left({ }^{*}\right)$ holds for $d^{\prime}=p q$. Similarly, I can write the latter inequality as $\frac{\pi_{\bar{p} q}}{\pi_{p \bar{q}}}>\left(\frac{a}{1-a}\right)^{n_{p \bar{q}}^{\mathbf{v}}-n \bar{p} q}$ since $n_{p}^{\mathbf{v}}-n_{q}^{\mathbf{v}}=\left(n-n_{\bar{p} q}^{\mathbf{v}}\right)-\left(n-n_{p \bar{q}}^{\mathbf{v}}\right)=n_{p \bar{q}}^{\mathbf{v}}-n_{\bar{p} q}^{\mathbf{v}}$. Rearranging it, I obtain

$$
\pi_{\bar{p} q} a^{n \overline{\bar{p}} q}(1-a)^{-n_{\bar{p} q}^{\mathbf{v}}}>\pi_{p \bar{q}} a^{n_{\bar{p} \bar{q}}^{\mathbf{v}}}(1-a)^{-n_{p \bar{q}}^{\mathbf{v}}} .
$$

As $\mathbf{v}=\mathbf{t}$, multiplying both sides of $(35)$ by $(1-a)^{n}$, I obtain $\pi_{\bar{p} q} a^{n_{\bar{p} q}^{\mathrm{t}}}(1-a)^{n-n_{\bar{p} q}^{\mathrm{t}}}>$ $\pi_{p \bar{q}} a_{p \bar{q}}^{n_{p}^{\mathrm{t}}}(1-a)^{n-n_{p \bar{q}}^{\mathrm{t}}}$ which by (25) and (26), is equivalent to $\left(^{*}\right)$ for $d^{\prime}=p \bar{q}$.

The proof for $d=p \bar{q}$ is analogous and follows from above arguments.

B. Conversely, assume informative voting is efficient under $f$. I consider any $\mathbf{v} \in \mathcal{J}^{n}$ and the corresponding type profile $\mathbf{t}=\mathbf{v}$. Since informative voting is efficient, the decision $d=f(\mathbf{v})$ is efficient for $\mathbf{t}(=\mathbf{v})$, i.e., $E(u(d, S) \mid \mathbf{t})>E\left(u\left(d^{\prime}, S\right) \mid \mathbf{t}\right)$ for all $d^{\prime} \in \mathcal{J} \backslash\{d\}$. Recal from part A that $n_{\bar{p} q}^{\mathbf{v}}=n-n_{p}^{\mathbf{v}}, n_{p \bar{q}}^{\mathbf{v}}=n-n_{q}^{\mathbf{v}}$, and $n_{p q}^{\mathbf{v}}=$ $n_{p}^{\mathbf{v}}+n_{q}^{\mathbf{v}}-n$.

Let $d=p q$. From $E(u(p q, S) \mid \mathbf{t})>E(u(\bar{p} q, S) \mid \mathbf{t})$, I can write $\pi_{p q} a^{n_{p}^{\mathbf{t}}+n_{q}^{\mathbf{t}}-n}(1-$ $a)^{2 n-n_{p}^{\mathrm{t}}-n_{q}^{\mathrm{t}}}>\pi_{\bar{p} q} a^{n-n_{p}^{\mathrm{t}}}(1-a)^{n_{p}^{\mathrm{t}}}$ using (24) and (25). Rearranging this inequality, I have $\frac{\pi_{p q}}{\pi_{\bar{p} q}}>\left(\frac{a}{1-a}\right)^{2 n-2 n_{p}^{\mathbf{t}}-n_{q}^{\mathbf{t}}}=\alpha\left(n_{p}^{\mathbf{t}}, n_{q}^{\mathbf{t}}\right)$. Thus, $d=p q \Longrightarrow \frac{\pi_{p q}}{\pi_{\bar{p} q}}>\alpha\left(n_{p}^{\mathbf{v}}, n_{q}^{\mathbf{v}}\right)$. One can analogously show that $d=p q \Longrightarrow \frac{\pi_{p q}}{\pi_{p \bar{q}}}>\alpha\left(n_{q}^{\mathbf{v}}, n_{q}^{\mathbf{v}}\right)$ from $E(u(p q, S) \mid \mathbf{t})>$ $E(u(p \bar{q}, S) \mid \mathbf{t})$ using $(24)$ and $(26)$.

Let $d=\bar{p} q$. From $E(u(p q, S) \mid \mathbf{t})<E(u(\bar{p} q, S) \mid \mathbf{t})$, I know that I already have $\frac{\pi_{p q}}{\pi_{\bar{p} q}}<$ $\alpha\left(n_{p}^{\mathbf{t}}, n_{q}^{\mathbf{t}}\right)=\alpha\left(n_{q}^{\mathbf{v}}, n_{p}^{\mathbf{v}}\right)$. From $E(u(\bar{p} q, S) \mid \mathbf{t})>E(u(p \bar{q}, S) \mid \mathbf{t})$, I can write $\pi_{\bar{p} q} a^{n-n_{p}^{\mathbf{t}}}(1-$ $a)^{n_{p}^{\mathrm{t}}}>\pi_{p \bar{q}} a^{n-n_{q}^{\mathrm{t}}}(1-a)^{n_{q}^{\mathrm{t}}}$. Rearranging, $\frac{\pi_{\bar{p} q}}{\pi_{p \bar{q}}}>\left(\frac{a}{1-a}\right)^{n_{p}^{\mathrm{t}}+n_{q}^{\mathrm{t}}}$. The RHS of the inequality 
is equal to $\beta\left(n_{p}^{\mathbf{t}}, n_{q}^{\mathbf{t}}\right)$, which is equal to $\beta\left(n_{p}^{\mathbf{v}}, n_{q}^{\mathbf{v}}\right)$ as $\mathbf{v}=\mathbf{t}$. Thus, $d=\bar{p} q \Longrightarrow \frac{\pi_{\bar{p} q}}{\pi_{p \bar{q}}}>$ $\beta\left(n_{p}^{\mathbf{v}}, n_{q}^{\mathbf{v}}\right)$.

Finally, the case of $d=p \bar{q}$ is straightforward since the converse of each of the two efficiency conditions $E(u(p \bar{q}, S) \mid \mathbf{t})>E(u(p q, S) \mid \mathbf{t})$ and $E(u(p \bar{q}, S) \mid \mathbf{t})>E(u(\bar{p} q, S) \mid \mathbf{t})$ has already been shown above.

Proof of Theorem 10. A. First, suppose $f: \mathcal{J}^{n} \rightarrow \mathcal{J}$ is a quota rule with exception with thresholds $m_{p}$ and $m_{q}$ and some exception rule, and suppose further that it makes informative voting efficient. I have to show that

(*) $m_{p}=h_{p}$ and $m_{q}=h_{q}$,

(**) $n_{r}^{\mathbf{v}}<h_{r}$ for each $r \in\{p, q\} \Longrightarrow f(\mathbf{v}) \in\{p \bar{q}, \bar{p} q\}$, and

$(* * *) \pi_{p q}>\pi_{\bar{p} q} \alpha\left(h_{p}, h_{q}\right)+\pi_{p \bar{q}} \alpha\left(h_{q}, h_{p}\right)$.

Consider a type profile $\mathbf{t} \in \mathcal{J}^{n}$. Assuming informative voting, the resulting voting profile $\mathbf{v}=\mathbf{t}$, thus, $n_{p}^{\mathbf{v}}=n_{p}^{\mathbf{t}}$ and $n_{q}^{\mathbf{v}}=n_{q}^{\mathbf{t}}$.

To show $\left(^{*}\right)$, suppose $n_{p}^{\mathbf{t}}=n$ and $n_{q}^{\mathbf{t}}=h_{q}$. By definition of $h_{q}, \pi_{p q}>\pi_{\bar{p} q} \alpha\left(n, h_{q}\right)+$ $\pi_{p \bar{q}} \alpha\left(h_{q}, n\right)$. Then, $f(\mathbf{v})=p q$ by Proposition 3. So, $h_{q} \geq m_{q}$ as $f$ is a quota rule with exception. Similarly, it can be shown that $h_{p} \geq m_{p}$. I now have to show the converse inequalities. Consider a voting profile $\mathbf{v} \in \mathcal{J}^{n}$ for which $n_{p}^{\mathbf{v}}=m_{p}$ and $n_{q}^{\mathbf{v}}=n$ which is greater than or equal to $m_{q}$. The resulting decision is $f(\mathbf{v})=p q$ by definition of $f$. Since $f$ makes informative voting with abstention efficient, $\pi_{p q}>$ $\pi_{\bar{p} q} \alpha\left(n_{p}^{\mathbf{v}}, n_{q}^{\mathbf{v}}\right)+\pi_{p \bar{q}} \alpha\left(n_{q}^{\mathbf{v}}, n_{p}^{\mathbf{v}}\right)=\pi_{\bar{p} q} \alpha\left(m_{p}, n\right)+\pi_{p \bar{q}} \alpha\left(n, m_{p}\right)$ by Proposition 3. Hence, $m_{p} \geq h_{p}$ by definition of $h_{p}$. Analogously, one shows that $m_{q} \geq h_{q}$. So, I have the first desired result $(*)$.

To show ${ }^{* *}$ ), let $n_{r}^{\mathbf{v}}<h_{r}$ for each $r \in\{p, q\}$ (note that if $h_{r}=0$ for any $r$, the premise of $(* *)$ is never satisfied, so this part of the proof can be omitted.) Under informative voting with abstention, $\mathbf{v}=\mathbf{t}$. By definition of $h_{p}$ and $h_{q}$, I must have $\pi_{p q}<\pi_{\bar{p} q} \alpha\left(n_{p}^{\mathbf{t}}, n_{q}^{\mathbf{t}}\right)+\pi_{p \bar{q}} \alpha\left(n_{q}^{\mathbf{t}}, n_{p}^{\mathbf{t}}\right)$ since the RHS is increasing in both arguments and $n_{r}^{\mathrm{t}}<h_{r}$ for each $r$. So, $p \bar{q}$ and $\bar{p} q$ are efficient, and as $f$ makes informative voting efficient, $f(\mathbf{v}) \in\{p \bar{q}, \bar{p} q\}$ by Proposition 3 .

Finally, I need to show (***). For any voting profile $\mathbf{v} \in \mathcal{J}^{n}$ for which $n_{p}^{\mathbf{v}}=h_{p}\left(=m_{p}\right)$ and $n_{q}^{\mathbf{v}}=h_{q}\left(=m_{q}\right)$, I have $f(\mathbf{v})=p q$ by definition of $f$, so that by Proposition 3 , $\pi_{p q}>\pi_{\bar{p} q} \alpha\left(n_{p}^{\mathbf{t}}, n_{q}^{\mathbf{t}}\right)+\pi_{p \bar{q}} \alpha\left(n_{q}^{\mathbf{t}}, n_{p}^{\mathbf{t}}\right)$, i.e., $\pi_{p q}>\pi_{\bar{p} q} \alpha\left(h_{p}, h_{q}\right)+\pi_{p \bar{q}} \alpha\left(h_{q}, h_{p}\right)$.

B. Conversely, let $\pi_{p q}>\pi_{\bar{p} q} \alpha\left(h_{p}, h_{q}\right)+\pi_{p \bar{q}} \alpha\left(h_{q}, h_{p}\right)$. I now show that the quota rule with exception $f$ with thresholds $h_{p}$ and $h_{q}$ and the exception rule (16) makes informative voting efficient. I start by proving that for all $k, l \in\{0, \ldots, n\}$,

$$
\pi_{p q}>\pi_{\bar{p} q} \alpha(k, l)+\pi_{p \bar{q}} \alpha(l, k) \Leftrightarrow\left[k \geq h_{p} \text { and } l \geq h_{q}\right] .
$$

If $k \geq h_{p}$ and $l \geq h_{q}$, then $\pi_{\bar{p} q} \alpha(k, l)+\pi_{p \bar{q}} \alpha(l, k) \leq \pi_{\bar{p} q} \alpha\left(h_{p}, h_{q}\right)+\pi_{p \bar{q}} \alpha\left(h_{q}, h_{p}\right)$, where the inequality holds because the expression is decreasing in each argument. If $k<h_{p}$, 
$\pi_{p q}<\pi_{\bar{p} q} \alpha(k, l)+\pi_{p \bar{q}} \alpha(l, k)$ which holds by definition of $h_{p}$. Analogously, if $l<h_{q}$, then $\pi_{p q}<\pi_{\bar{p} q} \alpha(k, l)+\pi_{p \bar{q}} \alpha(l, k)$.

Now consider any type profile $\mathbf{t} \in \mathcal{J}^{n}$ and assume informative voting. I must show that $f(\mathbf{v})$ is efficient for $\mathbf{t}$. First, if $n_{p}^{\mathbf{t}} \geq h_{p}$ and $n_{q}^{\mathbf{t}} \geq h_{q}$, the decision is $f(\mathbf{v})=p q$, which is efficient by Proposition 3 since $\pi_{p q}>\pi_{\bar{p} q} \alpha\left(n_{p}^{\mathbf{t}}, n_{q}^{\mathbf{t}}\right)+\pi_{p \bar{q}} \alpha\left(n_{q}^{\mathbf{t}}, n_{p}^{\mathbf{t}}\right)$ by (36). Second, if $n_{p}^{\mathbf{t}}<h_{p}$ or $n_{q}^{\mathbf{t}}<h_{q}$, the resulting decision $f(\mathbf{v})$ is in $\{\bar{p} q, p \bar{q}\}$, which is efficient by Proposition 3 since $\pi_{p q}<\pi_{\bar{p} q} \alpha\left(n_{p}^{\mathbf{t}}, n_{q}^{\mathbf{t}}\right)+\pi_{p \bar{q}} \alpha\left(n_{q}^{\mathbf{t}}, n_{p}^{\mathbf{t}}\right)$ by (36).

\section{References}

Ahn, D., Oliveros S. (2014) Condorcet jur(ies) theorem. Journal of Economic Theory 150: $841-851$

Ahn, D., Oliveros S. (2012) Combinatorial voting. Econometrica, 80(1): 89-141

Austen-Smith, D., Banks, J. (1996) Information aggregation, rationality, and the Condorcet jury theorem. American Political Science Review 90(1): 34-45

Austen-Smith, D., Feddersen, T. (2006) Deliberation, preference uncertainty and voting rules. American Political Science Review 100(2): 209-217

Barelli, P., Bhattacharya, S., Siga, L. (2018). Full information equivalence in large elections. Working paper

Blumrosen, L., Feldman, M. (2013), Mechanism design with a restricted action space. Games and Economic Behavior 82: 424-443.

Bovens, L., Rabinowicz, W. (2006) Democratic answers to complex questions: an epistemic perspective. Synthese 150(1): 131-153

Bozbay, I., Dietrich, F., Peters, H. (2014) Judgment aggregation in search for the truth. Games and Economic Behavior 87: 571-590

Bozbay, I., Peters, H. (2017) Information aggregation with continuum of types. Working paper

De Clippel G., Eliaz K. (2015) Premise versus outcome-based information aggregation. Games and Economic Behavior 89: 34-42

Dietrich, F. (2016) Judgment aggregation and agenda manipulation. Games and Economic Behavior 95: 113-136

Dietrich, F., List, C. (2007a) Strategy-proof judgment aggregation. Economics and Philosophy 23: 269-300

Dietrich, F., List, C. (2007b) Judgment aggregation by quota rules: majority voting generalized. Journal of Theoretical Politics 19(4): 391-424 
Dokow, E., Falik, D. (2012) Models of manipulation on aggregation of binary evaluations. In Preceedings of the Fourth International Workshop on Computational Social Choice, (pp. 191-202)

Feddersen, T., Pesendorfer, W. (1996) The Swing voter's curse. American Economic Review 86 (3): 408-424

Feddersen, T., Pesendorfer, W. (1997) Voting behavior and information aggregation in elections with private information. Econometrica 65 (5): 1029-1058

List, C. (2005) The probability of inconsistencies in complex collective decisions. Social Choice and Welfare 24(1): 3-32

List, C., Pettit, P. (2011) Group agency: The possibility, design and status of corporate agents. Oxford University Press

List, C., Polak, B. eds. (2010) Symposium on judgment aggregation, Journal of Economic Theory 145 (2)

McLennan, A. (1998) Consequences of the Condorcet jury theorem for beneficial information aggregation by rational agents. American Political Science Review 92 (2): $413-418$

Nehring, K., Puppe, C. (2002) Strategy-proof social choice on single-peaked domains: possibility, impossibility and the space between. Working paper, University of California at Davis

Nehring, K., Puppe, C. (2007) The structure of strategy-proof social choice. Part I: General characterization and possibility results on median spaces. Journal of Economic Theory 135: 269-305

Pivato, M. (2013) Voting rules as statistical estimators. Social Choice and Welfare 40(2): $581-630$

Schmitz, P. W., Tröger, T. (2012). The (sub-) optimality of the majority rule. Games and Economic Behavior 74(2): 651-665.

Van Zandt, T. (2007) Communication complexity and mechanism design. Journal of the European Economic Association 5: 543-553. 